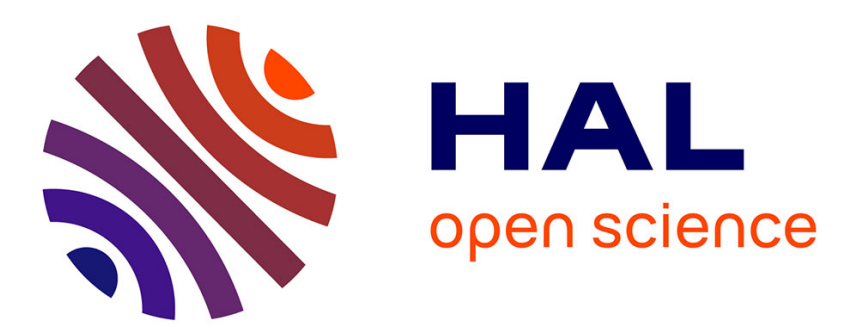

\title{
Collision avoidance in shared slots in wireless devices of the Internet of Things: models and simulations
}

\author{
Pascale Minet, Paul Mühlethaler, Ines Khoufi
}

\section{To cite this version:}

Pascale Minet, Paul Mühlethaler, Ines Khoufi. Collision avoidance in shared slots in wireless devices of the Internet of Things: models and simulations. Annals of Telecommunications - annales des télécommunications, 2018, 10.1007/s12243-018-0693-9 . hal-01957245

\section{HAL Id: hal-01957245 \\ https://hal.science/hal-01957245}

Submitted on 17 Dec 2018

HAL is a multi-disciplinary open access archive for the deposit and dissemination of scientific research documents, whether they are published or not. The documents may come from teaching and research institutions in France or abroad, or from public or private research centers.
L'archive ouverte pluridisciplinaire HAL, est destinée au dépôt et à la diffusion de documents scientifiques de niveau recherche, publiés ou non, émanant des établissements d'enseignement et de recherche français ou étrangers, des laboratoires publics ou privés. 


\title{
Collision Avoidance in Shared Slots in Wireless Devices of the Internet of Things: Models and Simulations
}

\author{
Pascale Minet*, Paul Muhlethaler*, Ines Khouf* \\ *Inria, 2 rue Simone Iff, CS 42112, 75589 Paris Cedex 12, France. Email: firstname.name@inria.fr
}

This is a pre-print of an article published in the Annals of Telecommunications, December 2018, pp 1-15. The final authenticated version is available online at: https://doi.org/10.1007/s12243-018-0693-9.

\begin{abstract}
In this paper we propose an analysis of a slot-based protocol designed for devices of the Internet of Things (IoT). In contrast to other TDMA-based protocols, this scheme uses a random technique to access shared slots, similarly to CSMA protocols. In practice, the transmissions are scheduled in a given back-off window of slots whose duration allows the transmission of a packet and its acknowledgment. Therefore this protocol can be analyzed according to the methodology introduced by Bianchi for the IEEE 802.11 protocol even if the protocol studied differs in many aspects. The model we use is also particular because we succeed in obtaining a Markov model even though the scheme used to send a packet (in a node) may depend on the transmission of the previous packet.

We distinguish two protocols; in the first one, at the initial stage or after a successful transmission, the packets are transmitted without any back-off, whereas in the second protocol each transmission is always preceded by the count down of a random back-off. Extensive simulations validate the both protocols models. In addition, the performances of these protocols are compared with those of slotted Aloha and a protocol using a constant backoff window.
\end{abstract}

\section{Index Terms}

Wireless networks, Model, Collision Avoidance, Shared slots, Medium Access, Markov Model, Slotted Aloha.

\section{CONTEXT AND MOTIVATION}

The Internet of Things (IoT) refers to billions of connected devices able to sense their environment and exchange data. These devices are smart items (e.g. a connected watch), autonomous vehicles, smart buildings, etc. The goal of these devices is to improve our daily life. For instance, they help to monitor our health and assist elderly people [1], increase road safety by assisting maneuvers for lane changing [2], [3], decrease the energy consumed by homes, and detect air pollution [4].

In addition, we are currently entering the fourth industrial revolution with Industrie 4.0 [5], [6]. The first one came about through the introduction of mechanical production facilities using water and steam power. The second revolution was made thanks to the introduction of labor division and mass production with the help of electrical energy. In the third one, electronic and IT (Information Technologies) systems have been massively used to further automate production. Currently, the real transformation is going to be through the use of cyber-physical systems. The complexity degree constantly increases with each new revolution. With the fourth revolution [5], we are witnessing a greater convergence between operational technology and information technology, through the use of the Industrial Internet of Things (IoT).

IoT relies on wireless networks supporting data gathering applications. Data gathering applications have strong requirements in terms of latency, throughput, robustness and power autonomy. The question is: which wireless sensor network technology is able to support such requirements. Most wireless sensor networks deployed up to now have used a technology based on the IEEE 802.15.4 standard [7]. Time Slotted Channel Hopping (TSCH) is specified in amendment e of the IEEE 802.15.4 standard [8]. It is based on a time slotted medium access operating on several channels in parallel. Frequency hopping is used to protect against perturbations. TSCH has been designed for process automation, process control, equipment monitoring and more generally the Internet of Things. In TSCH, all transmissions are scheduled in time and space: they take place in a pair (time slot, channel offset). Each channel offset is mapped on a physical channel. This mapping changes at each time slot. Frequency hopping contributes to reducing the effects of multipath and interferences. Two types of slots are distinguished: dedicated and shared. In a dedicated slot, only the transmitter is allowed to transmit, whereas the receiver(s) is awake to receive the transmission and the other nodes are sleeping in order to save energy. The absence of collisions in the dedicated slots leads to a higher throughput and a higher power autonomy of nodes. Dedicated slots are used to collect application data. In shared slots, all nodes are awake and all nodes that have a message to transmit are allowed to transmit. As a consequence, a collision occurs each time there is more than one transmitter in the same time slot and on the same channel. For unicast messages, the transmitter is aware of a possible collision, because it has not received an acknowledgment. In such a condition, the message is 
retransmitted, unless the maximum number of transmissions has already been reached. Notice that for broadcast and multicast messages, the transmitter does not know the existence of the collision.

Shared slots are generally used for network control and network adaptivity to topology or application changes. It would be too expensive in terms of bandwidth and latency to reserve dedicated slots for such messages that are not frequent. That is why shared slots that multiplex the transmitters are favored.

In this paper, we propose to model various methods of collision avoidance on shared slots in a wireless slotted network. All the methods studied in this paper are fully distributed and based on a probabilistic transmission by each network node. Two methods will be studied and modeled, including the method used by TSCH. These models will be validated through simulations on the NS3 simulation tool [9]. Performance of these methods are compared with the well-known slotted Aloha method [10].

\section{RELATED WORK AND OUR CONTRIBUTION}

This section is organized in three parts. The first part deals with various collision avoidance methods used in wireless networks. In the second part, we focus on how these methods have been modeled to derive the normalized throughput of the network. In both parts, we position our contributions with regard to this related work. The third part presents the organization of this paper.

\section{A. Probabilistic methods for collision avoidance in wireless networks}

All the methods studied in this paper are fully distributed and based on a probabilistic transmission by each network node. Probabilistic collision avoidance methods can be classified into three categories, according to their assumptions:

- The number of competing nodes is known. It has been proven that the normalized throughput is maximized when the transmission probability of each node is equal to $1 / N$, where $N$ is the number of competitors. This is the method used in slotted Aloha [10]. When $N$ becomes large, the normalized throughput tends to $1 / e$, which can be approximated by 0.3679. This is illustrated in Figure 1, where Ptrans denotes the node transmission probability.

- Each node continuously follows the channel state. Such methods require each node to permanently listen to the channel, which is energy consuming.

- Each node uses backoffs only. A backoff is a random value drawn in a backoff window defined by its low value and its high value. Such methods are easy to implement and do not require additional synchronization among nodes.

Consequently, the only probabilistic collision avoidance methods that do not consume much energy and do not rely on additional assumptions are those based on backoffs. These are the methods studied in this paper. More precisely, each node draws a random backoff in a backoff window. This backoff is decremented at each slot. The node waits until the backoff is zero to be allowed to transmit.

The methods vary in the backoff window parameters:

- the size: it may be constant or increased with the number of successive collisions.

- the reset: after each transmission, or only after each successful transmission, or at each first transmission of a message, etc.

- the increment: for instance, after each unsuccessful transmission of a message, until a maximum value is reached.

\section{B. Models for collision avoidance methods in wireless networks}

The first studies dealing with collision avoidance in wireless slotted networks were done for the IEEE 802.11 wireless network. The seminal paper is Bianchi's paper [11]. The analytical model based on a Markov chain designed in this paper is still in use, even if many extensions have been brought, as in, for instance, [12]. The first computation of the normalized throughput is given, assuming a finite number of wireless nodes and ideal channel conditions.

More recently, the authors of [13] show that when some CSMA/CA backoff parameters (e.g. the initial backoff, the backoff multiplier and the number of retries) are different from the standard, some unfairness may occur in single-hop 802.11 networks with saturated transmitters.

Focusing on the slotted access of the IEEE 802.15.4 protocol, we can cite the studies [14], [15], [16], [17] that provide an evaluation of the throughput, average delay, energy consumption and reliability. All these papers propose nearly the same Markov chain model. In contrast to the present paper, the back-off evolves during the lifetime of the packet when it encounters collisions. However, when the packet is rejected (i.e. the maximum number of transmissions of this message has been reached and the last transmission collides) the protocol does not remember the actual value of the collision window for the next transmission as our model does. Moreover the models in [14], [15], [16], [17] handle the case where the node buffers always have pending packets. Our model handles the more realistic situation where we have a given packet generation rate in a single buffer with loss and provides a much simpler normalization equation than [14], [15], [16], [17]. 


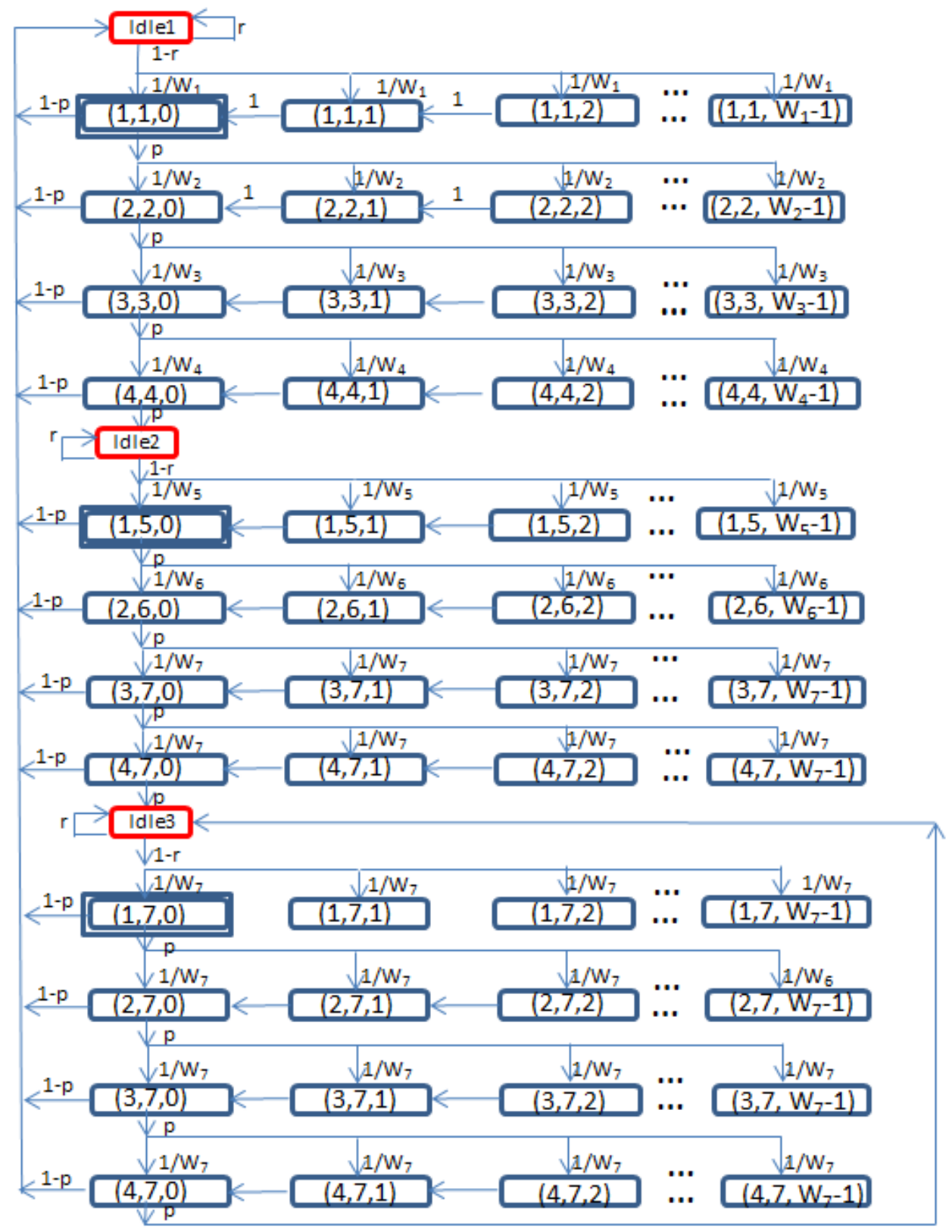

Fig. 1. Normalized throughput with slotted Aloha.

\section{Paper organization}

This paper is organized as follows. In Section III, we define the problem of shared slots access; the notation used throughout the paper is given. In Section IV, we analyze the medium access protocol that always performs a backoff before each transmission. We propose a node Markov model taking into account the backoff stage and the maximum number of message transmissions. We evaluate the probabilities of success, collision and empty slots. This model is validated through simulations done with NS3. We then model the protocol that does not perform a backoff before the first transmission of each message and design its Markov model in Section V, which is also validated by means of NS3 simulations. We evaluate the performance of each protocol in terms of successful message transmissions, rejected messages and node fairness in Section VI. We also compare these proocols with slotted Aloha and a protocol using a constant backoff window. Finally, we conclude in Section VII.

\section{Problem statement}

\section{A. Assumptions}

In this paper, we adopt the following assumptions: 
- $\left.A_{1}\right)$ We consider a wireless slotted network: each transmission starts at the beginning of a slot. The slot size allows the transmission of one packet and its immediate acknowledgment when this packet is sent point-to-point.

- $\left.A_{2}\right)$ All the slots are shared.

- $A_{3}$ ) Access to the shared slots is ruled by a backoff expressed in terms of the number of shared slots that the competing node must wait for before being allowed to transmit.

- $\left.A_{4}\right)$ There is no channel sensing before transmitting. In a wireless slotted network where all nodes are synchronized to start their transmission at the beginning of a slot like in $\mathrm{TSCH}$, it would be useless to assess a clear channel state before transmitting, because all nodes would perform it simultaneously. Finding the channel clear, they would all transmit simultaneously leading to a collision. In addition, channel clear assessment consumes energy. As a consequence, it is not performed in the wireless slotted network considered.

- $\left.A_{5}\right)$ There are $N$ nodes in the network and all these nodes are assumed to be 1-hop neighbors.

- $\left.A_{6}\right)$ Each node generates a message with a probability $1-r$.

- $\left.A_{7}\right)$ Each node has a buffer which can contain at most one message: if a message is generated, when the node's buffer is not empty, it is lost.

\section{B. Evaluation criteria and Notation}

We want to select the collision avoidance method that:

- Maximizes the normalized throughput that is measured as the probability of successful transmission

- Minimizes the probability of message rejection. A message that has not been acknowledged after the maximum number of transmissions is rejected.

- Ensures node fairness. For this purpose, we use the Jain fairness index [18] on the node opportunity to transmit. We have

$$
J\left(T x_{1}, \ldots, T x_{n}\right)=\frac{\left(\sum_{i=1}^{n} T x_{i}\right)^{2}}{n \sum_{i=1}^{n} T x_{i}^{2}}
$$

where $T x_{i}$ denotes the number of transmission attempts of node $i$. The Jain fairness index belongs to the interval $[0,1]$. The closer the Jain fairness index is to 1 , the better the node fairness is.

- Is the least energy-consuming. Since the sensor nodes have a limited amount of energy, this energy should be reserved for useful transmissions.

We now introduce some notations that will be used in this paper that are summarized in Table I.

TABLE I

NOTATIONS

\begin{tabular}{|c|c|c|}
\hline \multicolumn{3}{|c|}{ Constants } \\
\hline Name & Meaning & Default value in $\mathrm{TSCH}$ \\
\hline$N$ & Number of neighboring nodes & $\in[4,32]$ \\
\hline$R$ & Maximum number of transmissions & 4 \\
\hline $\operatorname{Min} B$ & Minimum backoff stage & 1 \\
\hline $\operatorname{Max} B$ & Maximum backoff stage & 7 \\
\hline$W_{j}$ & $\begin{array}{l}\text { Upper bound of backoff window } \\
\text { at stage } j\end{array}$ & $\begin{array}{l}W_{j}=2^{j} \\
\operatorname{Min} B \leq j \leq \operatorname{Max} B\end{array}$ \\
\hline \multicolumn{3}{|l|}{ States } \\
\hline Name & Meaning & Default value \\
\hline$(i, j, k)$ & $\begin{array}{l}\text { State of the wireless node considered } \\
i \text { is the number of transmissions } \\
j \text { is the backoff stage } \\
k \text { is the number of slots to wait for }\end{array}$ & $\begin{array}{l}1 \leq i \leq R \\
\operatorname{Min} B \leq j \leq \operatorname{Max} B \\
0 \leq k \leq W_{j}-1\end{array}$ \\
\hline \multicolumn{3}{|c|}{ Probabilities } \\
\hline Name & Meaning & Default value \\
\hline$p$ & $\begin{array}{l}\text { Probability of a collision when } \\
\text { a node is transmitting }\end{array}$ & \\
\hline$\tau$ & Node transmission probability & \\
\hline$r$ & Probability of staying Idle & $1-1 / \mathrm{N}$ \\
\hline
\end{tabular}

\section{Simulation}

The NS3 simulation tool is used to evaluate the performance of different collision avoidance methods (in shared slots). It also validates the models proposed. 
Let $S$ denote the total number of slots and $E$ be the number of empty slots. Simulation results allow us to compute the values of $S$ and $E$ and more generally, the values of the different probabilities given in Table II.

TABLE II

COMPUTATION OF PROBABILITIES

\begin{tabular}{lll}
\hline Name & Meaning & Value \\
\hline$\tau$ & $\begin{array}{l}\text { Probability for a node to transmit } \\
\text { in a random slot }\end{array}$ & $\frac{\text { Total number of transmissions }}{S}$ \\
$P_{\text {success }}$ & $\begin{array}{l}\text { Probability for a random slot to } \\
\text { correspond to a successful trans- } \\
\text { mission }\end{array}$ & $\frac{\text { Number of slots with successful transmission }}{S}$ \\
$P_{\text {empty }}$ & $\begin{array}{l}\text { Probability for a random slot to be } \\
\text { empty }\end{array}$ & $\frac{\text { Number of empty slots }}{S}$ \\
$P_{\text {collide }}$ & $\begin{array}{l}\text { Probability for a random slot to } \\
\text { correspond to a collision }\end{array}$ & $\frac{\text { Number of slots with 2 or more transmitters }}{S}$ \\
\hline
\end{tabular}

Each result is the average of 30 simulation runs. In each simulation run, 10000 slots are simulated. The parameters given in Table I are used: the maximum number of transmissions of a message is 4 and the backoff stage belongs to the interval $[1,7]$ and $W_{j}=2^{j}$.

\section{BACKOFF BEFORE EACH TRANSMISSION}

We first describe the collision avoidance method performing a backoff systematically before each transmission.

\section{A. Principle of the method}

Each node in the slotted wireless network behaves as follows.

- Before transmitting a message, a backoff is drawn in the backoff window. The backoff window increases with the backoff stage. The backoff stage $j$ starts with 1 and is incremented after each unsuccessful transmission (see the definition below) up to a maximum value $\operatorname{Max} B$. The backoff window at stage $j$ is equal to $\left[0, W_{j}-1\right]$. The backoff drawn in this window represents the number of shared slots the node must wait for before being allowed to transmit its message.

- When a message is sent, two cases are possible:

- either an acknowledgment is received and the transmission is considered successful. The backoff stage is reset to the initial value $\operatorname{Min} B$. The node serves the next message in its Transmit queue.

- no acknowledgment is received after a time-out and the transmission is considered unsuccessful. There are two possible cases:

* the node has already transmitted this message $R$ times, this message is rejected, a failure notification is reported to the upper layer. Notice that the backoff stage is incremented by one (up to the maximum value MaxB).

* otherwise, the node increments its backoff stage $j$ by one (up to the maximum value $M a x B$ ) and its number of transmissions for this message. It then draws a random backoff in $\left[0, W_{j}-1\right]$. When the backoff has elapsed, it transmits its message.

- The message is removed from the buffer only after its acknowledgment has been received or the maximum number of retries has been reached.

\section{B. Model based on a Markov chain}

Idle denotes the node state with an empty buffer. Any message generated while the node is not in the Idle state is lost. Except in the Idle state where the buffer of the node is empty, the state of the node considered is represented by a triplet $(i, j, k)$ where $i$ denotes the number of transmissions of the current message considered, $j$ denotes the backoff stage and $k$ is the backoff drawn in the backoff window $\left[0, W_{j}-1\right]$. We have the following inequalities:

- $1 \leq i \leq R$, where $R$ denotes the maximum number of transmissions of a message.

- $\operatorname{Min} B \leq j \leq \operatorname{MaxB}$, where $\operatorname{Min} B$ and $\operatorname{Max} B$ are constant parameters fixed by the MAC protocol considered.

- $i \leq j$

- $0 \leq k \leq W_{j}-1$.

Such a node behavior can be modeled by a Markov chain. This model, following in the footsteps of Bianchi [11] has many differences:

- There is no Clear Channel Assess (CCA) before transmitting a message.

- The backoff is decremented at each shared slot even if this slot is not empty. 
- The backoff stage after $R$ unsuccessful transmissions of a message is not reset to the initial value for the next message. As a consequence, the first transmission of a new message may correspond to three different states, which we explain for the case $R=4$ and $\operatorname{Max} B=7$ :

- $(1,1,0)$, when the previous message transmission was successful. The backoff stage is equal to 1 .

- $(1,5,0)$, when the previous message was rejected. The backoff stage is equal to $R+1=5$.

- $(1,7,0)$, when the two previous messages were rejected. The backoff stage is equal to its maximum value i.e. 7 .

- Medium saturation is not assumed. We suppose that the probability of packet generation is $1-r$ at each slot.

Let us focus on the Idle state, state of a node when it has no message to transmit. We distinguish three Idle states according to the result of the previous transmission. These states are denoted:

- $I d l e_{1}$, when the previous message transmission was successful. The backoff stage will be reset to 1 for the next transmission.

- $I d l e_{2}$, when the previous message was rejected. The backoff stage will be incremented for the next transmission, it will be $R+1=5$.

- $I d l e_{3}$, when the two previous messages were rejected. The backoff stage keeps its current maximum value of 7 .

The introduction of these states allows us to model the node behavior by a Markov chain, where the next node state depends only on the current state and the event occurring. The Markov chain of this contention avoidance method is depicted in Figure 2, where the three Idle states are depicted by a red rectangle, whereas the three states corresponding to the first message transmission are depicted by a blue double-lined rectangle.

When a node transmits, its transmission collides with a probability $p$.

To improve the readability of the notation we denote by:

- $a_{i}$ the probability of being in state $(i, i, 0)$ for $i=1,2,3$ or $4=R$,

- $b_{i}$ the probability of being in state $(i, \min (4+i, 7), 0)$ for $i=1,2,3$ or $4=R$,

- $c_{i}$ the probability of being in state $(i, 7,0)$ for $i=1,2,3$ or $4=R$, with $\operatorname{Max} B=7<2 * R$,

- $I_{1}$ the probability of being in state $I d l e_{1}$,

- $I_{2}$ the probability of being in state $I d l e_{2}$,

- $I_{3}$ the probability of being in state $I_{d l e_{3}}$,

- $\tau$ the probability that a node transmits in a random slot.

We obtain the following system of equations:

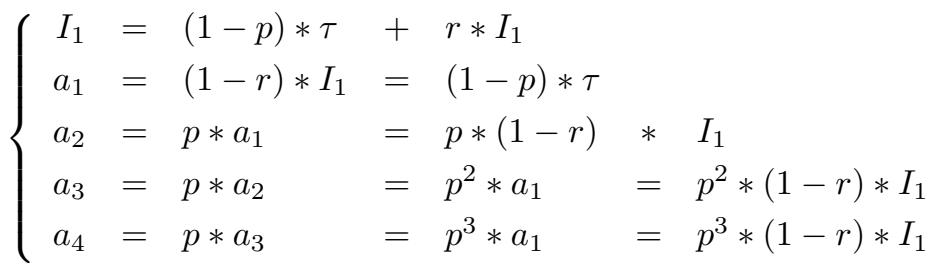

$$
\begin{aligned}
& \begin{cases}I_{2}=p * a_{4} & +r * I_{2} \\
b_{1}=(1-r) * I_{2} & =p * a_{4} \quad=p^{4} * a_{1} \\
b_{2}=p * b_{1} & =p^{5} * a_{1} \\
b_{3}=p * b_{2} & =p^{6} * a_{1} \\
b_{4}=p * b_{3} & =p^{7} * a_{1}\end{cases} \\
& \begin{cases}I_{3}=p *\left(b_{4}+c 4\right) & +r * I_{3} \\
c_{1}=(1-r) * I_{3} & =p *\left(b_{4}+c_{4}\right) \\
c_{2}=p * c_{1} & \\
c_{3}=p * c_{2} & =p^{2} * c_{1} \\
c_{4}=p * c_{3} & =p^{3} * c_{1} \\
c_{1}=p * b_{4} & +p * c_{4}\end{cases}
\end{aligned}
$$




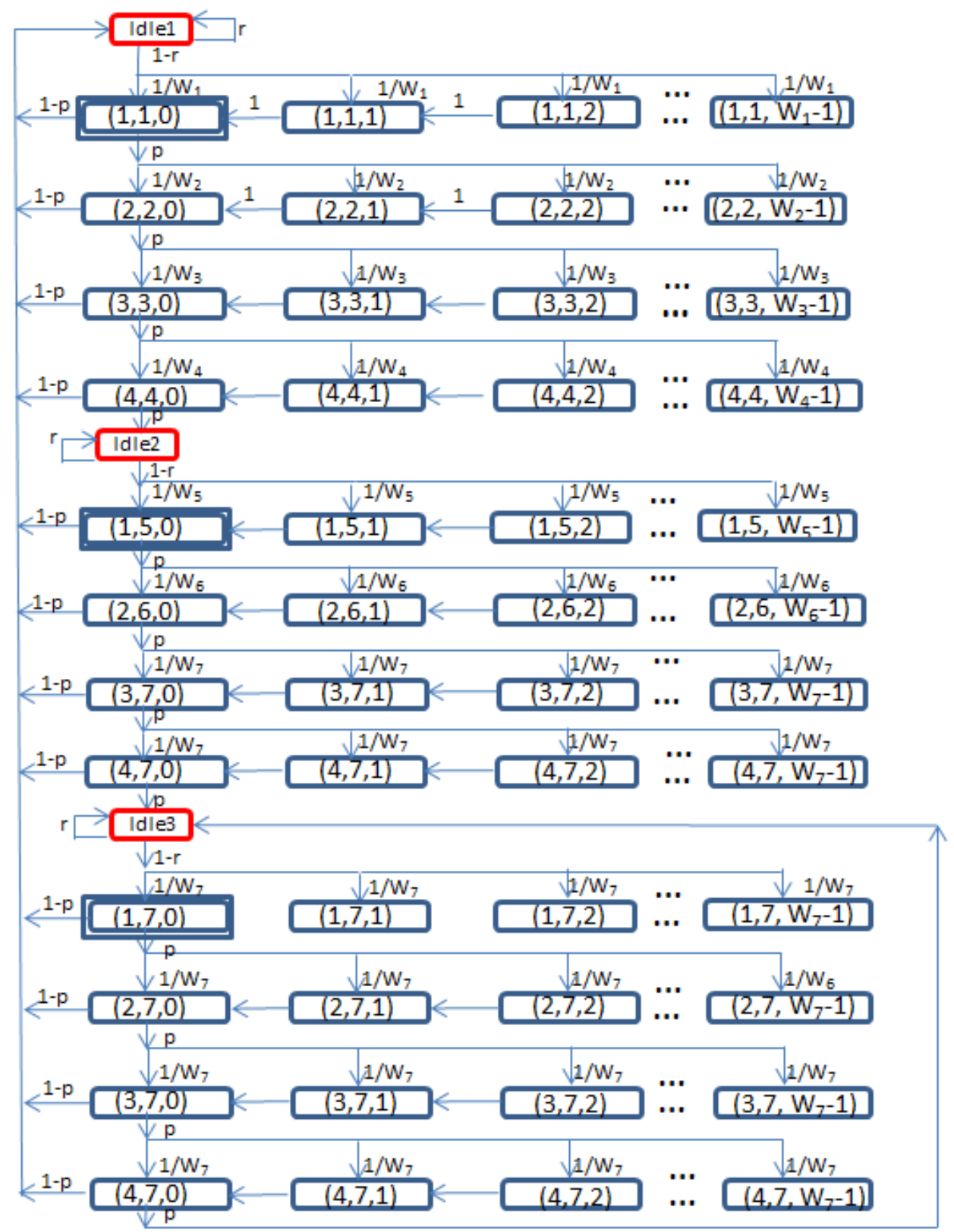

Fig. 2. The Markov chain of the collision avoidance method on shared slots with a backoff before each transmission.

We get:

$c_{1} \stackrel{p * b_{4}}{=}+p^{4} * c_{1}$

$c_{1} *\left(1-p^{4}\right)=p * b_{4}=p^{8} * a_{1}$

$c_{1}=\frac{p^{8} * a_{1}}{1-p^{4}}$

If we now focus on the different backoff states before the first transmission of a message generated while in state $I_{d l e_{1}}$, we have:

$$
\left\{\begin{aligned}
a_{1, W_{1}-1} & =a_{1} / W_{1} \\
a_{1, W_{1}-2} & =2 * a_{1} / W_{1} \\
a_{1,0} & =W_{1} * a_{1} / W_{1}
\end{aligned}\right.
$$


Summing member to member, we get:

$$
\left\{\begin{array}{l}
\sum_{k=0}^{W_{1}-1} a_{1, k}=a_{1} *\left(W_{1}+1\right) / 2 \\
\sum_{k=0}^{W_{2}-1} a_{2, k}=a_{2} *\left(W_{2}+1\right) / 2=p * a_{1} *\left(W_{2}+1\right) / 2 \\
\sum_{k=0}^{W_{3}-1} a_{3, k}=a_{3} *\left(W_{3}+1\right) / 2=p^{2} * a_{1} *\left(W_{3}+1\right) / 2 \\
\sum_{k=0}^{W_{4}-1} a_{4, k}=a_{4} *\left(W_{4}+1\right) / 2=p^{3} * a_{1} *\left(W_{4}+1\right) / 2
\end{array}\right.
$$

Similarly, we obtain:

$$
\left\{\begin{array}{l}
\sum_{k=0}^{W_{5}-1} b_{1, k}=b_{1} *\left(W_{5}+1\right) / 2=p^{4} * a_{1} *\left(W_{5}+1\right) / 2 \\
\sum_{k=0}^{W_{6}-1} b_{2, k}=b_{2} *\left(W_{6}+1\right) / 2=p^{5} * a_{1} *\left(W_{6}+1\right) / 2 \\
\sum_{k=0}^{W_{7}-1} b_{3, k}=b_{3} *\left(W_{7}+1\right) / 2=p^{6} * a_{1} *\left(W_{7}+1\right) / 2 \\
\sum_{k=0}^{W_{7}-1} b_{4, k}=b_{4} *\left(W_{7}+1\right) / 2=p^{7} * a_{1} *\left(W_{7}+1\right) / 2
\end{array}\right.
$$

and

$$
\left\{\begin{array}{l}
\sum_{k=0}^{W_{7}-1} c_{1, k}=c_{1} *\left(W_{7}+1\right) / 2=\frac{p^{8}}{1-p^{4}} * a_{1} *\left(W_{7}+1\right) / 2 \\
\sum_{k=0}^{W_{7}-1} c_{2, k}=c_{2} *\left(W_{7}+1\right) / 2=\frac{p^{9}}{1-p^{4}} * a_{1} *\left(W_{7}+1\right) / 2 \\
\sum_{k=0}^{W_{7}-1} c_{3, k}=c_{3} *\left(W_{7}+1\right) / 2=\frac{p^{10}}{1-p^{4}} * a_{1} *\left(W_{7}+1\right) / 2 \\
\sum_{k=0}^{W_{7}-1} c_{4, k}=c_{4} *\left(W_{7}+1\right) / 2=\frac{p^{11}}{1-p^{4}} * a_{1} *\left(W_{7}+1\right) / 2
\end{array}\right.
$$

For simplicity reasons, we set $A=\sum_{i=1}^{4} \sum_{k=0}^{W_{i}-1} a_{i, k}$, $B=\sum_{i=1}^{4} \sum_{k=0}^{W_{\min (i+4,7)}-1} b_{i, k}$ and $C=\sum_{i=1}^{4} \sum_{k=0}^{W_{7}-1} c_{i, k}$. We have:

$$
\begin{aligned}
& \left\{\begin{aligned}
A= & a_{1} * \frac{W_{1}+1}{2}+p * a_{1} * \frac{W_{2}+1}{2}+p^{2} * a_{1} * \frac{W_{3}+1}{2} \\
& +p^{3} * a_{1} * \frac{W_{4}+1}{2} \\
B= & p^{4} * a_{1} * \frac{W_{5}+1}{2}+p^{5} * a_{1} * \frac{W_{6}+1}{2}+p^{6} * a_{1} * \frac{W_{7}+1}{2} \\
& +p^{7} * a_{1} * \frac{W_{7}+1}{2}
\end{aligned}\right. \\
& C= \\
& \begin{cases}A= & \left(1+p+p^{2}+p^{3}\right) *\left(\frac{p^{8} * a_{1}}{1-p^{4}}\right) * \frac{W_{7}+1}{2}=a_{1} * \frac{p^{8}}{1-p} * \frac{W_{7}+1}{2} \\
B= & a_{1} *\left(\frac{W_{1}+1}{2}+p * \frac{W_{2}+1}{2}+p^{2} * \frac{W_{3}+1}{2}+p^{3} * \frac{W_{4}+1}{2}\right) \\
C= & \frac{p^{8} * a_{1}}{1-p} * \frac{W_{7}+1}{2}\end{cases}
\end{aligned}
$$

From the model depicted in Figure 2 and normalizing the sum of the probabilities, we get:

$$
\left\{\begin{aligned}
\tau & =a_{1}+a_{2}+a_{3}+a_{4}+b_{1}+b_{2}+b_{3}+b_{4}+c_{1}+c_{2}+c_{3}+c_{4} \\
1 & =A+B+C+I_{1}+I_{2}+I_{3} \\
I_{1} & =\frac{a_{1}}{1-r} \\
I_{2} & =\frac{p^{4} * a_{1}}{1-r} \\
I_{3} & =\frac{p^{8} * a_{1}}{(1-r) *\left(1-p^{4}\right)}
\end{aligned}\right.
$$

By replacing the $a_{i}, b_{i}$ and $c_{i}$ and $I_{i}$ by their value function of $a_{1}$ and replacing $A+B+C$ by its value computed previously, we have:

$$
\begin{aligned}
& \left\{\begin{aligned}
\tau= & a_{1} *\left(1+p+p^{2}+p^{3}+p^{4}+p^{5}+p^{6}+\frac{p^{7}}{1-p}\right) \\
1= & a_{1} *\left(\frac{W_{1}+1}{2}+p * \frac{W_{2}+1}{2}+p^{2} * \frac{W_{3}+1}{2}+p^{3} * \frac{W_{4}+1}{2}\right. \\
& +p^{4} * \frac{W_{5}+1}{2}+p^{5} * \frac{W_{6}+1}{2}+p^{6} * \frac{W_{7}+1}{2} \\
& \left.+\frac{p^{7}}{1-p} * \frac{W_{7}+1}{2}+\frac{1}{1-r}+\frac{p^{4}}{1-r}+\frac{p^{8}}{(1-r) *\left(1-p^{4}\right)}\right)
\end{aligned}\right. \\
& \left\{\begin{aligned}
\tau= & \frac{a_{1}}{1-p} \\
a_{1}= & \left(\frac{W_{1}+1}{2}+p * \frac{W_{2}+1}{2}+p^{2} * \frac{W_{3}+1}{2}+p^{3} * \frac{W_{4}+1}{2}\right. \\
& +p^{4} * \frac{W_{5}+1}{2}+p^{5} * \frac{W_{6}+1}{2}+p^{6} * \frac{W_{7}+1}{2} \\
& \left.+\frac{p^{7}}{1-p} * \frac{W_{7}+1}{2}+\frac{1}{1-r}+\frac{p^{4}}{1-r}+\frac{p^{8}}{(1-r) *\left(1-p^{4}\right)}\right)^{-1}
\end{aligned}\right.
\end{aligned}
$$


By replacing $a_{1}$ by its value as a function of $p$, we have:

$$
\begin{aligned}
\tau= & \frac{1}{1-p} \\
* & \left(\frac{W_{1}+1}{2}+p * \frac{W_{2}+1}{2}+p^{2} * \frac{W_{3}+1}{2}+p^{3} * \frac{W_{4}+1}{2}+p^{4} * \frac{W_{5}+1}{2}\right. \\
& +p^{5} * \frac{W_{6}+1}{2}+p^{6} * \frac{W_{7}+1}{2}+\frac{p^{7}}{1-p} * \frac{W_{7}+1}{2}+\frac{1}{1-r}+\frac{p^{4}}{1-r} \\
& \left.+\frac{p^{8}}{(1-r) *\left(1-p^{4}\right)}\right)^{-1}
\end{aligned}
$$

We can compute $p$ the probability of a collision knowing that one node already transmits. It is equal to 1 minus the probability that none of the $N-1$ other nodes transmits. Hence, we obtain:

$$
p=1-(1-\tau)^{N-1}
$$

Replacing $p$ by its value as a function of $\tau$ in Equation 2 leads to $f(\tau)=\tau$ where the function $f($.$) is defined as follows:$

$$
\begin{aligned}
f(\tau)= & \frac{1}{(1-\tau)^{N-1}} \\
* & \left(\frac{W_{1}+1}{2}+\frac{W_{2}+1}{2} *\left(1-(1-\tau)^{N-1}\right)\right. \\
& +\frac{W_{3}+1}{2} *\left(1-(1-\tau)^{N-1}\right)^{2}+\frac{W_{4}+1}{2} *\left(1-(1-\tau)^{N-1}\right)^{3} \\
& +\frac{W_{5}+1}{2} *\left(1-(1-\tau)^{N-1}\right)^{4}+\frac{W_{6}+1}{2} *\left(1-(1-\tau)^{N-1}\right)^{5} \\
& +\frac{W_{7}+1}{2} *\left(1-(1-\tau)^{N-1}\right)^{6}+\frac{W_{7}+1}{2} * \frac{\left(1-(1-\tau)^{N-1}\right)^{7}}{(1-\tau)^{N-1}} \\
& \left.+\frac{1}{1-r}+\frac{\left(1-(1-\tau)^{N-1}\right)^{4}}{1-r}+\frac{\left(1-(1-\tau)^{N-1}\right)^{8}}{(1-r) *\left(1-\left(1-(1-\tau)^{N-1}\right)^{4}\right)}\right)^{-1}
\end{aligned}
$$

In the interval $[0,1]$, the function $f(\tau)-\tau$ is monotonic. Hence, there exists a unique solution $\tau \in[0,1)$ giving $f(\tau)=\tau$.

As an example, we compute the solution of $f(\tau)=\tau$ for the case $N=8$, assuming $r=1-1 / N$ and taking the default values adopted by TSCH and given in Table I. The value of $\tau$ solution of $f(\tau)=\tau$ is depicted in Figure 3, where $\tau \approx 0.1053$.

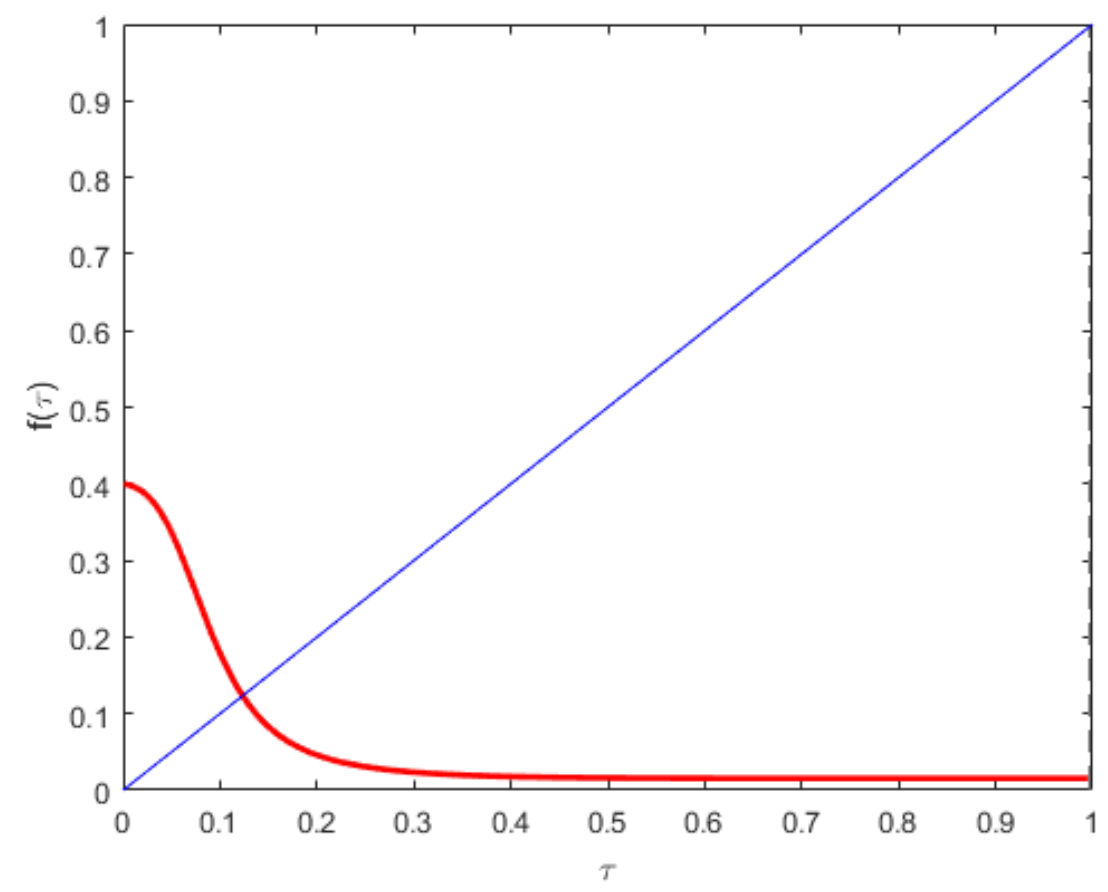

Fig. 3. The transmission probability $\tau=0.1053$ solution of $f(\tau)=\tau$ for $N=8$. 
After having computed the value of $\tau$, the unique solution of Equation 4, we can compute the probabilities of success, collision and empty slots as follows.

\section{Model validation by simulation}

$$
\begin{cases}a_{1} & =(1-p) * \tau \\ I_{1} & =a_{1} /(1-r)=\frac{(1-p) * \tau}{1-r} \\ I_{2} & =\frac{p^{4} * a_{1}}{1-r} \\ I_{3} & =\frac{p^{8} * a_{1}}{1-r} \\ P_{\text {success }} & =N * \tau *(1-\tau)^{N-1} \\ P_{\text {empty }} & =(1-\tau)^{N} \\ P_{\text {collide }} & =1-P_{\text {success }}-P_{\text {empty }}\end{cases}
$$

Assuming a backoff before each transmission, Figure 4 compares the results of simulations and the model for $4,8,16$ or 32 nodes, when $r=1-1 / N$. The values of Psuccess, also called normalized throughput, and Pempty are depicted. Since the value of Pcollide is deduced from the two previous, it is not depicted. Our Markov model is validated by the very close results obtained by simulation.

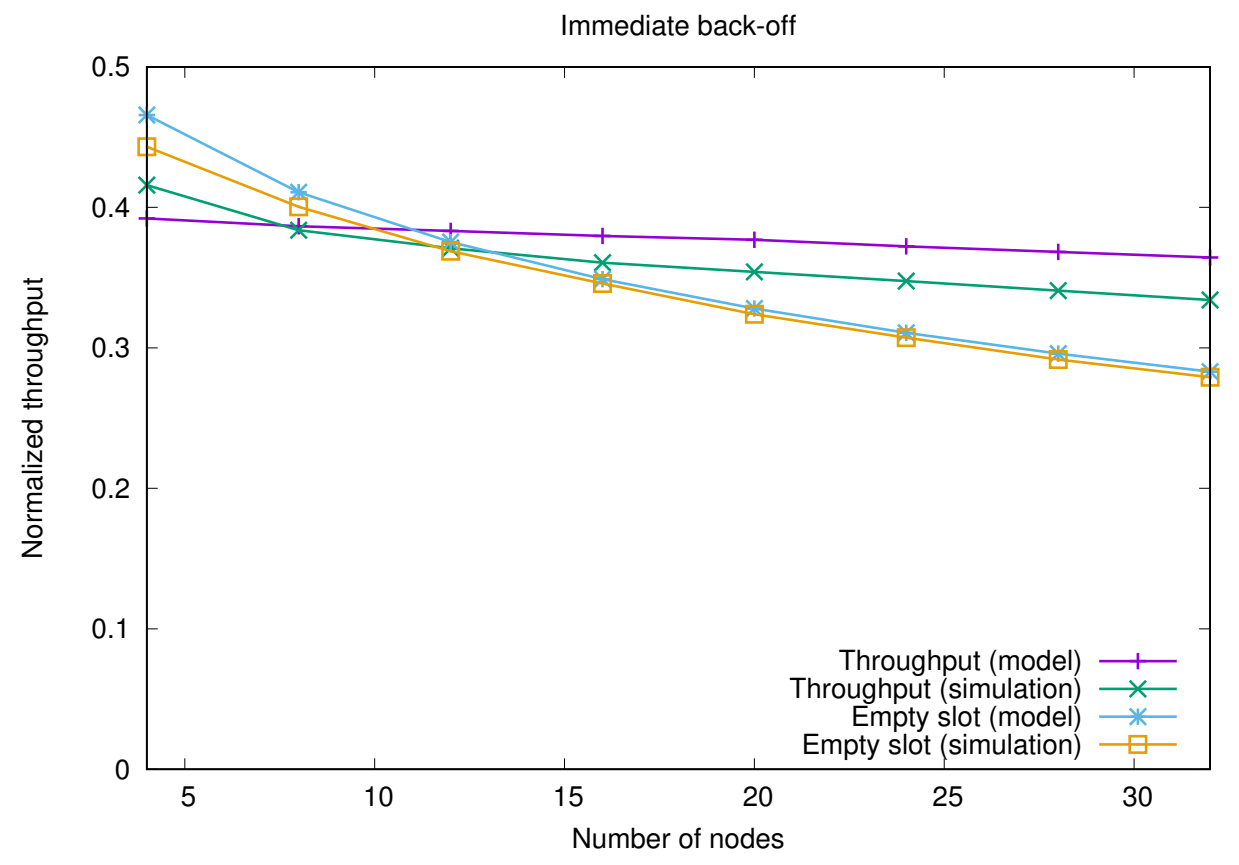

Fig. 4. Model validation.

\section{NO BACKOFF FOR THE FIRST TRANSMISSION}

We now focus on the collision avoidance method used by TSCH on the shared slots. It differs from the method explained in Section IV by the fact that no backoff is drawn before the first transmission of the message sent after the successful transmission of the previous message. It is also the case for the first transmission of the first message sent by the wireless node.

\section{A. Model based on a Markov chain}

The node behavior can be modeled by a Markov chain. As previously stated, the node state with an empty buffer is denoted Idle. Any message generated while the node is not in the Idle state is lost. Except in the Idle state where the buffer of the node is empty, the node state is represented by a triplet $(i, j, k)$ where $i$ denotes the number of transmissions of the current message, $j$ denotes the backoff stage and $k$ is the backoff drawn by the node in the backoff window $\left[0, W_{j}-1\right]$. We have the following inequalities:

- $1 \leq i \leq R$, where $R$ denotes the maximum number of transmissions of a message.

- $\operatorname{Min} B \leq j \leq \operatorname{Max} B$, where $\operatorname{MinB}$ and $\operatorname{Max} B$ are constant parameters fixed by the MAC protocol considered.

- $0 \leq k \leq W_{j}-1$. 
As a consequence, the first transmission of a new message may correspond to three different states, which we explain for the case $R=4$ and $\operatorname{Max} B=7$ :

- $(1,0,0)$, when the previous message transmission was successful. By convention, the backoff stage is set to 0 .

- $(1,4,0)$, when the previous message was rejected. The backoff stage is equal to $R=4$.

- $(1,7,0)$, when the two previous messages were rejected. The backoff stage is equal to its maximum value, i.e. $M a x B=7$.

Notice that unlike the Markov model presented in Section IV-B, the message transmission number is no longer less than or equal to the backoff stage, because in this collision avoidance method, the success or failure of the two previous messages determines the backoff stage of the current message. This explains the three different states corresponding to the first transmission of a message in the Markov model. These states are depicted by a blue double-lined rectangle in Figure 5, whereas the three Idle states are depicted by a red rectangle.

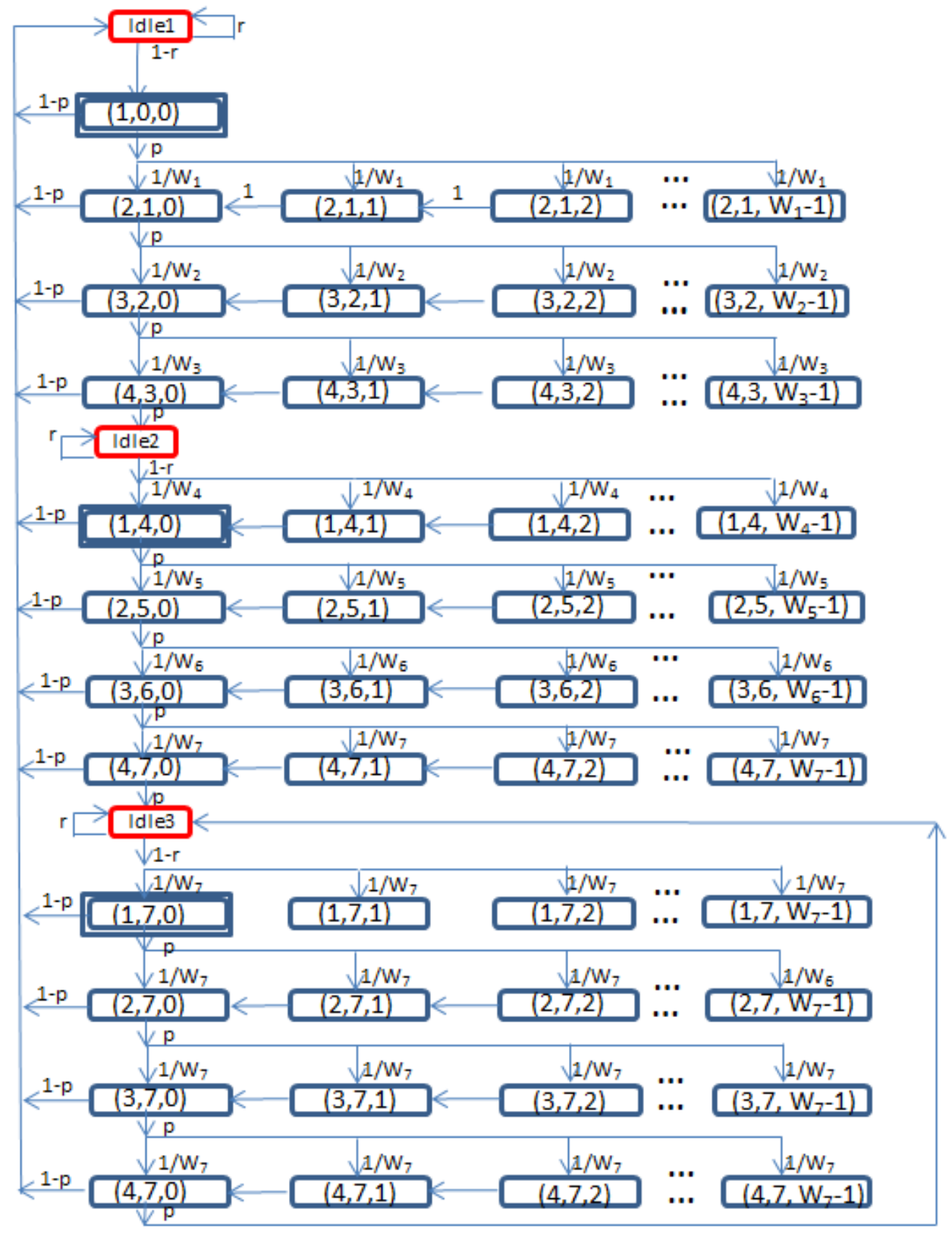

Fig. 5. The Markov chain of the collision avoidance method on shared slots of TSCH.

We get the same system of equations for $I_{1}, I_{2}$ and $I_{3}$ as in Section IV-B. By similarity with the analysis given in Section IV-B, we finally get: 


$$
\begin{aligned}
f(\tau)= & \frac{1}{(1-\tau)^{N-1}} \\
* \quad & \left(1+\left(1-(1-\tau)^{N-1}\right) * \frac{W_{1}+1}{2}+\left(1-(1-\tau)^{N-1}\right)^{2} * \frac{W_{2}+1}{2}\right. \\
& +\left(1-(1-\tau)^{N-1}\right)^{3} * \frac{W_{3}+1}{2}+\left(1-(1-\tau)^{N-1}\right)^{4} * \frac{W_{4}+1}{2} \\
& +\left(1-(1-\tau)^{N-1}\right)^{5} * \frac{W_{5}+1}{2}+\left(1-(1-\tau)^{N-1}\right)^{6} * \frac{W_{6}+1}{2} \\
& +\frac{\left(1-(1-\tau)^{N-1}\right)^{7}}{(1-\tau)^{N-1}} * \frac{W_{7}+1}{2}+\frac{1}{1-r} \\
& \left.+\frac{\left(1-(1-\tau)^{N-1}\right)^{4}}{1-r}+\frac{\left(1-(1-\tau)^{N-1}\right)^{8}}{(1-r) *\left(1-\left(1-(1-\tau)^{N-1}\right)^{4}\right)}\right)^{-1}
\end{aligned}
$$

In the interval $[0,1]$, the function $f(\tau)-\tau$ is monotonic. Hence, there exists a unique solution $\tau \in[0,1)$ giving $f(\tau)=\tau$.

To compare with the collision avoidance method presented in Section IV, we compute the solution of $f(\tau)=\tau$ for the case $N=8$, assuming $r=1-1 / N$ and taking the default values adopted by TSCH and given in Table I. The value of $\tau$ solution of $f(\tau)=\tau$ is depicted in Figure 6 , where $\tau \approx 0.1200$. This is a higher value than this found by the method performing always a backoff before each transmission which was $\tau \approx 0.1053$.

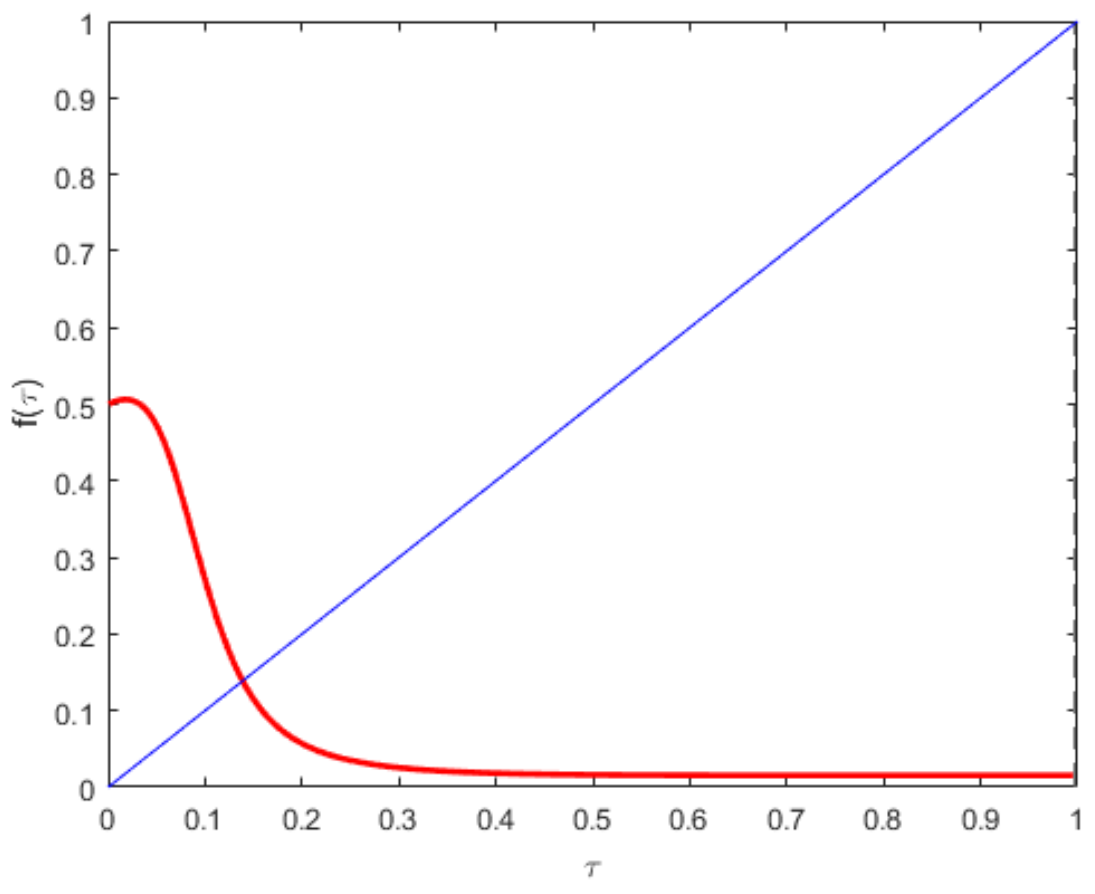

Fig. 6. The transmission probability $\tau=0.1200$ solution of $f(\tau)=\tau$ for $N=8$.

After having computed the value of $\tau$, the unique solution of $f(\tau)=\tau$, we can compute the probabilities of success, collision and empty slots as in Section IV.

\section{B. Model validation by simulation}

Assuming no backoff before the first transmission, Figure 7 compares the results obtained by simulations and the model for 4,8,16 or 32 nodes, when $r=1-1 / N$. The values of Psuccess, also called normalized throughput, and Pempty are depicted. Since the value of Pcollide is deduced from the two previous, it is not depicted. Our Markov model is validated by the very close results obtained by simulation.

\section{COMPARATIVE PERFORMANCE EVALUATION}

We now compare the two protocols, for a number of neighboring nodes $N$ belonging to [4,32]. We distinguish two cases of channel load: moderate and heavy. 


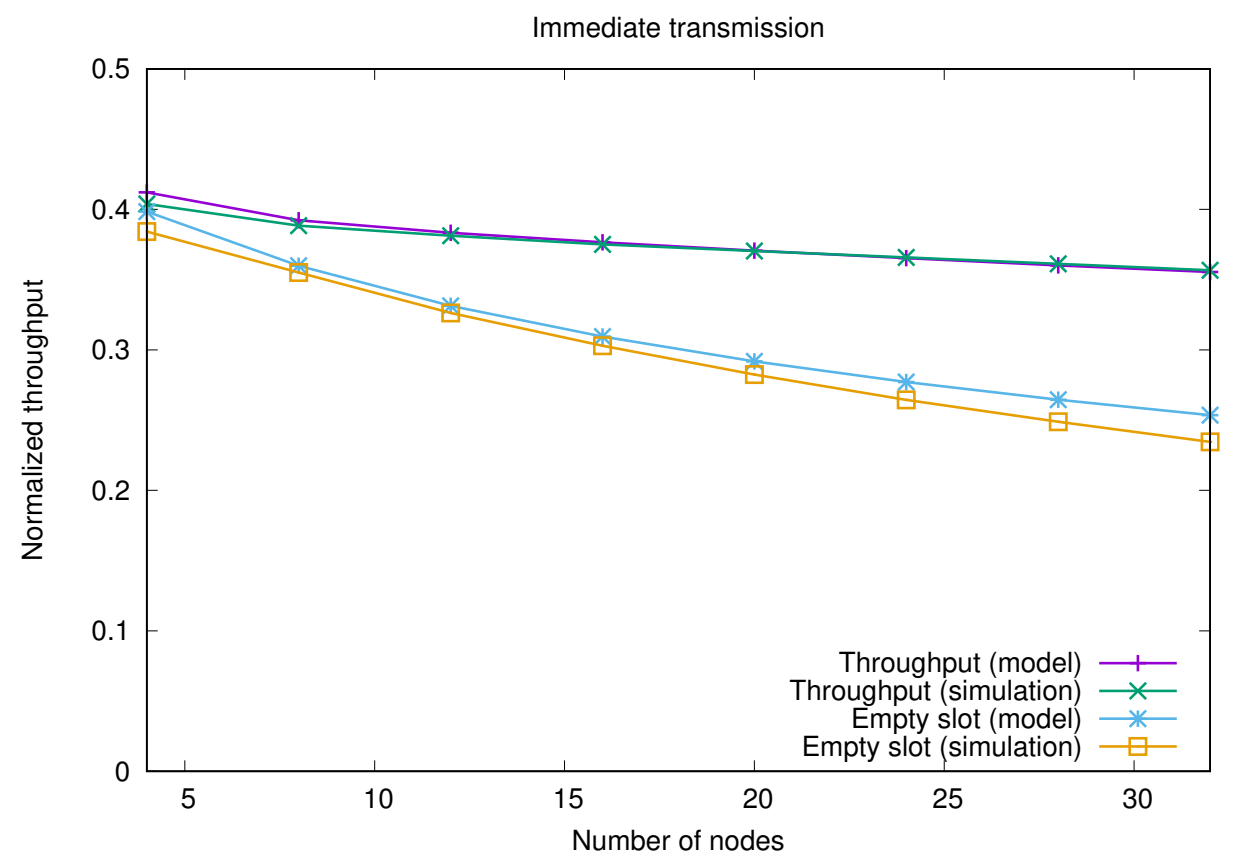

Fig. 7. Model validation.

\section{A. Moderately loaded medium}

When the medium is moderately loaded, simulation results concerning the probability of success, collision and empty slots are very stable, the error bars of the simulation for a confidence interval of $95 \%$ are very small and not visible in the figures. Figure 8 shows that for a number of neighboring nodes in $[1,7]$ the protocol used by TSCH provides a higher probability of success leading to a higher normalized throughput. This is no longer the case as soon as $N \geq 8$. Similarly, simulation results show a higher collision number, whatever the number of nodes. This is due to the fact that the first transmission of any message very often leads to a collision. The probability of empty slots that measures the channel under-utilization is minimized by TSCH.

In this comparison, we have adopted $r=1-1 / N$ or in other words the packet generation rate is $1 / N$ for each node and in each slot. Other values for this generation rate are acceptable and lead to a model well in accordance with simulation results. However, if the total load becomes too high, significant unfairness appears in the throughput for the TSCH protocol, as we will see in the next subsection. When the unfairness becomes high, the model is no longer able to predict the simulation results accurately.

\section{B. Highly loaded medium}

We now assume that each network node always has at least one message to transmit. This leads to a highly loaded medium and even to medium saturation. At medium saturation, we are interested in the normalized throughput, the ratio of successfully transmitted messages over the total number of messages that are either successfully transmitted or rejected, as well as node fairness. Node fairness is evaluated by the Jain fairness index as defined in Section III-B.

1) Results for TSCH: Table III provides the results obtained by TSCH in terms of normalized throughput, probability of empty slots, probability of collision in a slot, ratio of successful transmissions, for a number of nodes $N$ ranging from 2 to 32. Notice that the throughput obtained by TSCH is very good, especially for small values of $N$. However, this excellent performance is obtained at the cost of some unfairness. This unfairness is maximized for $N=2$. In such a scenario, we get simulation traces where the fairness is equal to 0.8371 only. We notice that in 10000 slots, 7441 transmission opportunities have been granted to node 1, compared with only 2896 transmission opportunities for node 2 . As a consequence, 6821 messages from node 1 have been successfully delivered against only 2226 messages for node 2 . This is reflected by the poor value of the Jain fairness index. The reason for this unfairness is the following: the node that succeeds in transmitting its message, immediately transmits its next message, whereas other nodes are waiting for the end of their backoff, that may reach 127 slots.

2) Results for slotted Aloha: Table IV depicts the results obtained by the simulation with NS3 of slotted Aloha for $N$ ranging from 4 to 32 neighbors. Simulation results are very close to the theoretical ones. We observe that Throughput = Psuccess $\rightarrow 1 / e$, when $N$ increases, as proved in [10]. For any number of nodes, the fairness provided by this method is 


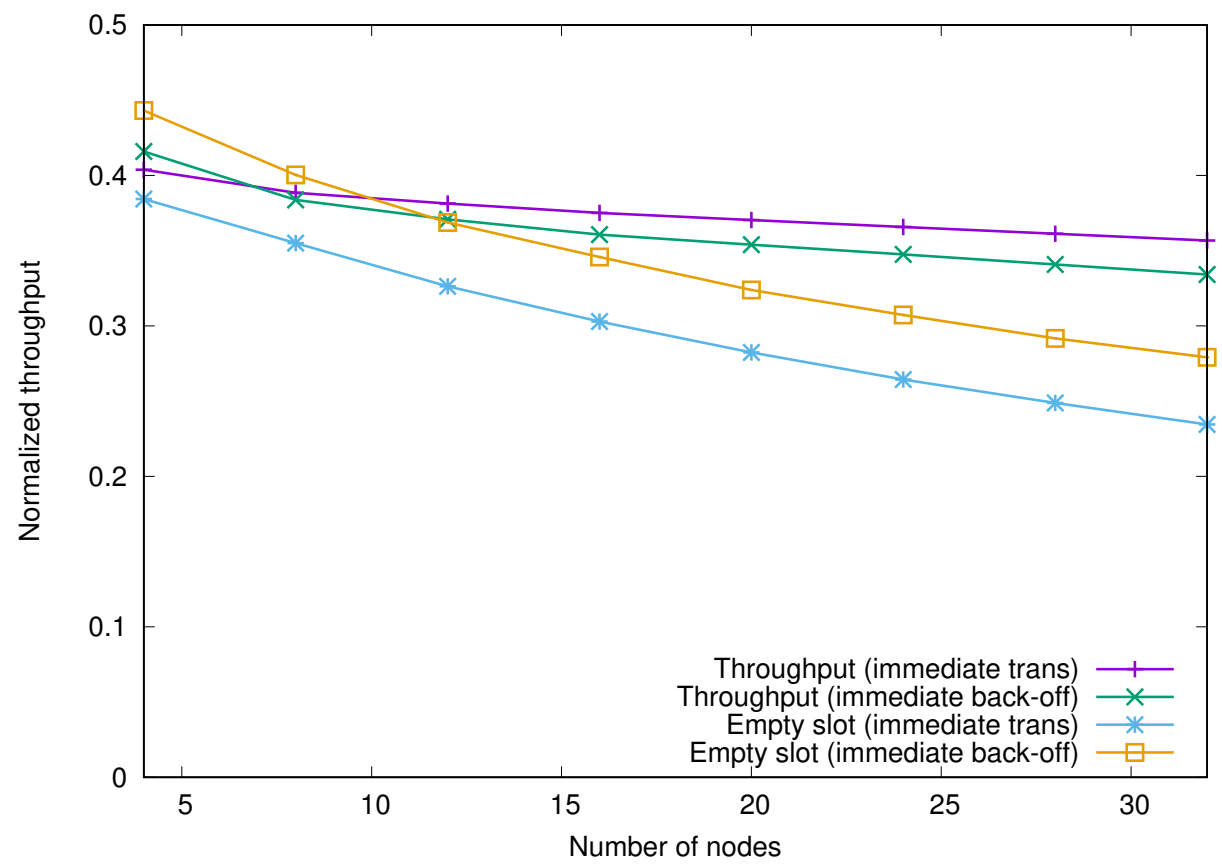

Fig. 8. Normalized throughput and probability of empty slots.

TABLE III

RESULTS OBTAINED BY TSCH.

\begin{tabular}{|c|c|c|c|c|c|c|}
\hline$N$ & Throughput & Pempty & Pcollide & Prejection & $\frac{\text { Psuccess }}{\text { Psuccess+Prejection }}$ & Fairness \\
\hline \hline 2 & 0.91156 & 0.0292 & 0.05928 & 0.01820 & 0.9818 & 0.9578 \\
4 & 0.7682 & 0.0737 & 0.1581 & 0.0589 & 0.9410 & 0.9614 \\
8 & 0.5795 & 0.1167 & 0.3039 & 0.1552 & 0.8448 & 0.9720 \\
16 & 0.4265 & 0.1279 & 0.4456 & 0.3061 & 0.6939 & 0.9716 \\
32 & 0.3166 & 0.107 & 0.5765 & 0.4901 & 0.5099 & 0.9808 \\
\hline
\end{tabular}

excellent and the probability of rejecting a message, because the maximum number of transmissions is reached without any success, is kept lower than $15 \%$. In this protocol, each node receives a fair share of the bandwidth equal to $1 / N$. Notice that for 32 nodes, this collision avoidance method provides a better throughput than TSCH.

TABLE IV

SiMULATION RESUlts OF SLOTTED ALOHA FOR DIFFERENT NUMBERS OF COMPETITORS.

\begin{tabular}{|c|c|c|c|c|c|c|}
\hline$N$ & Throughput & Pempty & Pcollide & Prejection & $\frac{\text { Psuccess }}{\text { Psuccess+Prejection }}$ & Fairness \\
\hline \hline 4 & 0.4227 & 0.3160 & 0.2613 & 0.1107 & 0.8893 & 0.9997 \\
8 & 0.3936 & 0.3422 & 0.2643 & 0.1356 & 0.8644 & 0.9993 \\
16 & 0.3806 & 0.2643 & 0.2643 & 0.1475 & 0.8525 & 0.9986 \\
32 & 0.3732 & 0.1356 & 0.2641 & 0.1533 & 0.8467 & 0.9969 \\
\hline
\end{tabular}

Figure 9 compares the performance of TSCH and the $1 / N$ Aloha protocol in terms of normalized throughput. For all values of $N$ in the range of 2 to 16, TSCH provides a higher throughput than $1 / N$ Aloha. For 32 nodes, slotted Aloha outperforms TSCH.

3) Results for Backoff before any transmission: Results obtained when the backoff is used before each transmission are given in Table V. The normalized throughput obtained by this method is less than that provided by TSCH for a number of nodes strictly less than 32 . For 32 nodes, the throughput obtained by this method and TSCH are very close. In all cases, it ensures a better fairness than TSCH, by reducing the probability of transmitting in the next slot for the successful transmitter.

Figure 10 depicts the normalized throughput obtained by TSCH and a variant implementing a backoff before each transmission. For all values of $N$ strictly less than 32, TSCH provides a higher throughput than its variant applying a backoff before each transmission. For higher values, this method is better. 


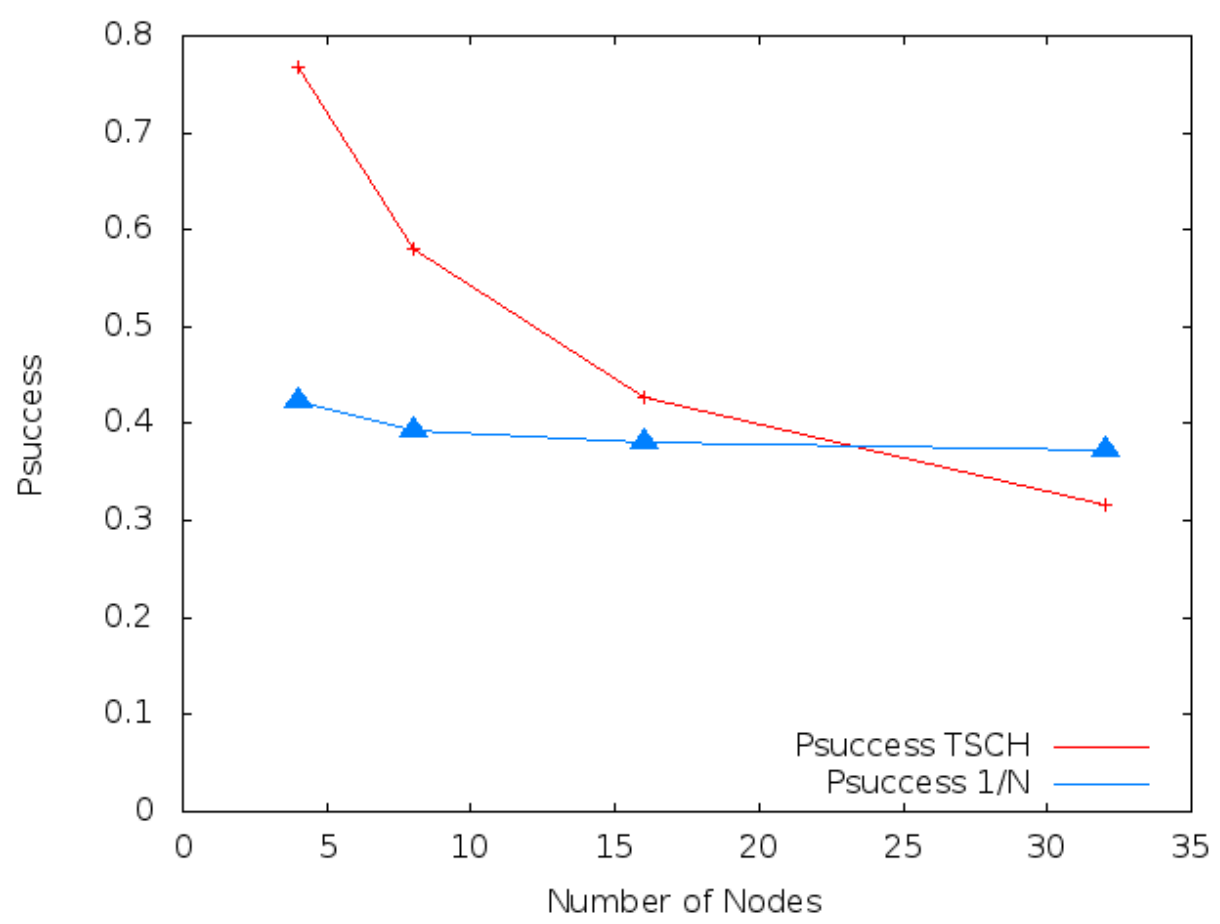

Fig. 9. Comparison between TSCH and $1 / N$ Aloha.

TABLE V

RESULTS OBTAINED BY THE PROTOCOL USING A BACKOFF BEFORE EACH TRANSMISSION.

\begin{tabular}{|c|c|c|c|c|c|c|}
\hline$N$ & Throughput & Pempty & Pcollide & Prejection & $\frac{\text { Psuccess }}{\text { Psuccess+Prejection }}$ & Fairness \\
\hline \hline 4 & 0.4765 & 0.3011 & 0.2224 & 0.1455 & 0.8545 & 0.9908 \\
8 & 0.4332 & 0.2546 & 0.3122 & 0.2538 & 0.7462 & 0.9882 \\
16 & 0.3807 & 0.2024 & 0.4170 & 0.3936 & 0.6064 & 0.9859 \\
32 & 0.3130 & 0.1412 & 0.5458 & 0.5625 & 0.4375 & 0.9873 \\
\hline
\end{tabular}

4) Results for Backoff before any transmission and a backoff window $W=2 * N$ : We now study a variant of the Backoffbefore-any-transmission method where the backoff window is constant and does not depend on the backoff stage. It is equal to $W=2 * N$. Table VI depicts the simulation results obtained. We observe that when $N$ increases, the performances provided by this method and slotted Aloha are very close in terms of normalized throughput and fairness, with a small advantage to slotted Aloha. However, this method provides a higher rejection probability than slotted Aloha. We also notice that compared with TSCH, it provides a higher fairness, because the node having successfully transmitted its message must draw a backoff before transmitting its next message.

TABLE VI

RESULTS OBTAINED BY A BACKOFF BEFORE EACH TRANSMISSION AND $W=2 * N$.

\begin{tabular}{|c|c|c|c|c|c|c|}
\hline$N$ & Throughput & Pempty & Pcollide & Prejection & $\frac{\text { Psuccess }}{\text { Psuccess }+ \text { Prejection }}$ & Fairness \\
\hline \hline 2 & 0.4443 & 0.4444 & 0.1113 & 0.0315 & 0.9685 & 0.9999 \\
4 & 0.4092 & 0.4102 & 0.1806 & 0.1117 & 0.8883 & 0.9999 \\
8 & 0.3888 & 0.3901 & 0.2212 & 0.1768 & 0.8232 & 0.9998 \\
16 & 0.3779 & 0.3805 & 0.2416 & 0.2126 & 0.7874 & 0.9995 \\
32 & 0.3716 & 0.3749 & 0.2536 & 0.2342 & 0.7657 & 0.9990 \\
\hline
\end{tabular}

Figure 11 depicts the normalized throughput obtained by TSCH and a variant that implements a backoff before each transmission with a backoff size $W=2 * N$. For all values of $N$ in the range of 2 to 32 , TSCH provides a higher throughput than its variant applying a backoff before each transmission with $W=2 * N$, when the number of nodes is less than or equal to 16. For 32 nodes the backoff method with $W=2 * N$ provides the highest throughput.

5) Comparative performance evaluation of the four collision avoidance methods: Figures 12, 13, 14 and 15 allow us to compare the performance of the four collision avoidance methods studied in this paper, namely TSCH, Backoff before each 


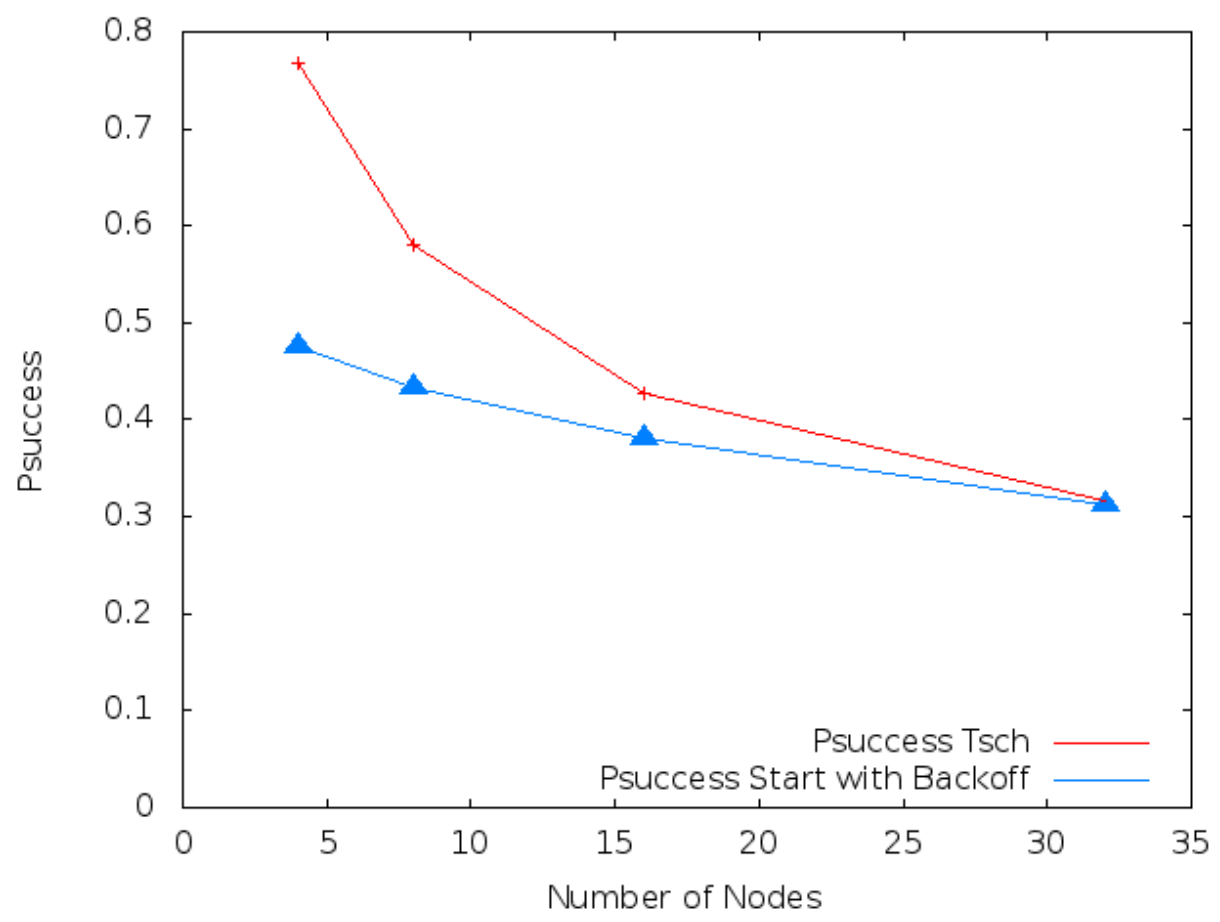

Fig. 10. Normalized throughput obtained by TSCH and a variant implementing a backoff before each transmission.

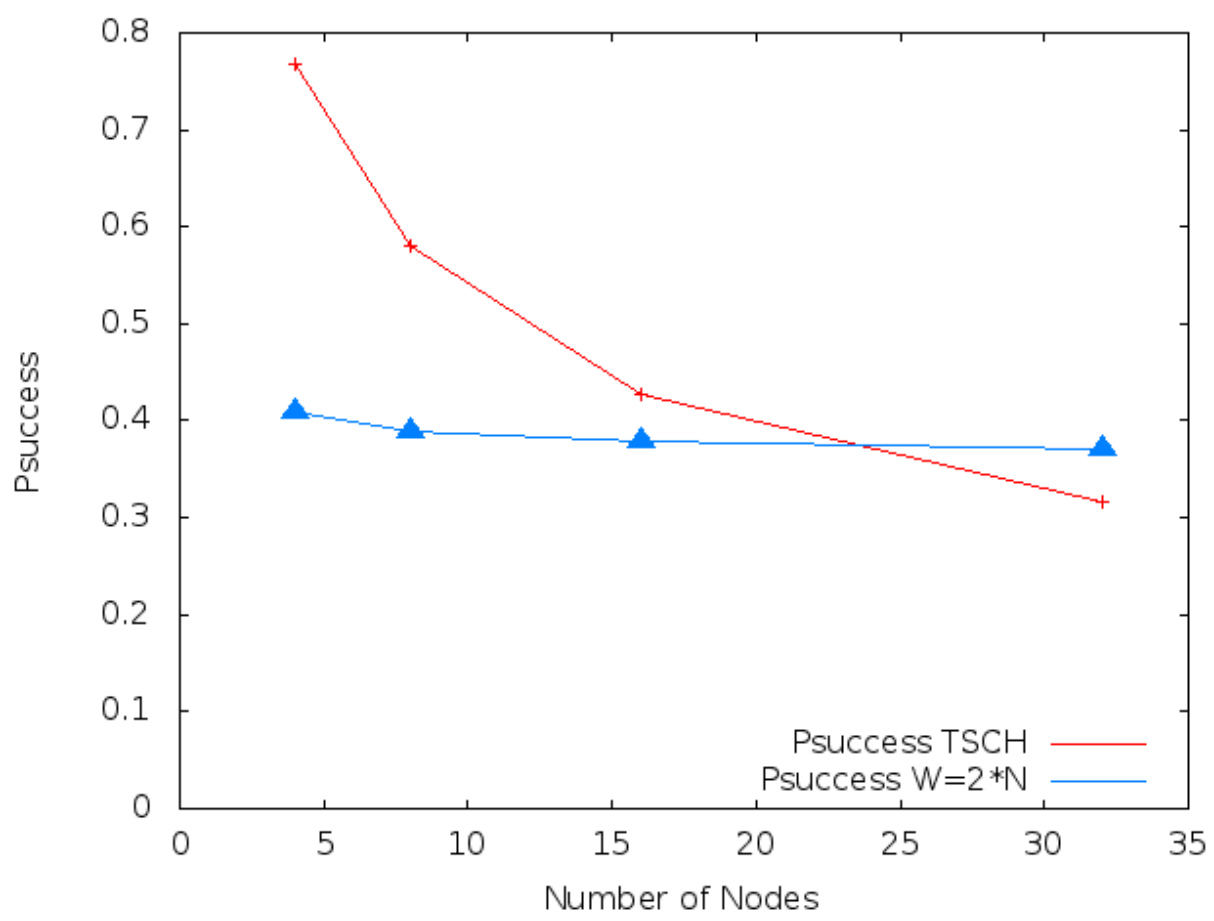

Fig. 11. Normalized throughput obtained by TSCH and a variant implementing a backoff before each transmission with $W=2 * N$.

transmission, slotted Aloha, Backoff before any transmission with a backoff window $W=2 * N$. The number of nodes varies from $4,8,16$ and 32 .

For all these values of $N$, the $1 / N$ Aloha protocol provides the best node fairness (higher than 0.9968 ) and the best ratio 
of successfully transmitted messages (higher than 0.8467). This is explained by the fact that each node receives a fair share (i.e. $1 / N$ ) of the bandwidth. We recall that in slotted Aloha each node transmits in each slot with a probability equal to $1 / N$.

The protocol used by TSCH provides the highest normalized throughput (higher than 0.4265 ) up to 16 nodes, the smallest ratio of empty slots (less than 0.1279 ), but the worst fairness (less than 0.9614 for 4 nodes). This is because the node that succeeds in transmitting its message, immediately transmits its next message, whereas other nodes are waiting for the end of their backoff. For 32 nodes, the best normalized throughput is obtained by slotted Aloha.

The protocol based on a backoff before each transmission provides a normalized throughput smaller than TSCH $(0.4332$ and 0.3807 for 8 and 16 nodes, respectively) but higher than the $1 / N$ Aloha protocol $(0,3927$ and 0.3806 for 8 and 16 nodes, respectively). For 32 nodes, the throughput becomes very close to that obtained by TSCH. It also ensures a better fairness than TSCH (higher than 0.986).

The protocol applying a backoff before each transmission with a backoff window $W=2 * N$ provides results that are very close to slotted Aloha. These results become closer when $N$ the number of competitors increases. However, the normalized throughput as well as the ratio of delivered messages are higher for slotted Aloha. Similarly, slotted Aloha provides the smallest probability of message rejection.

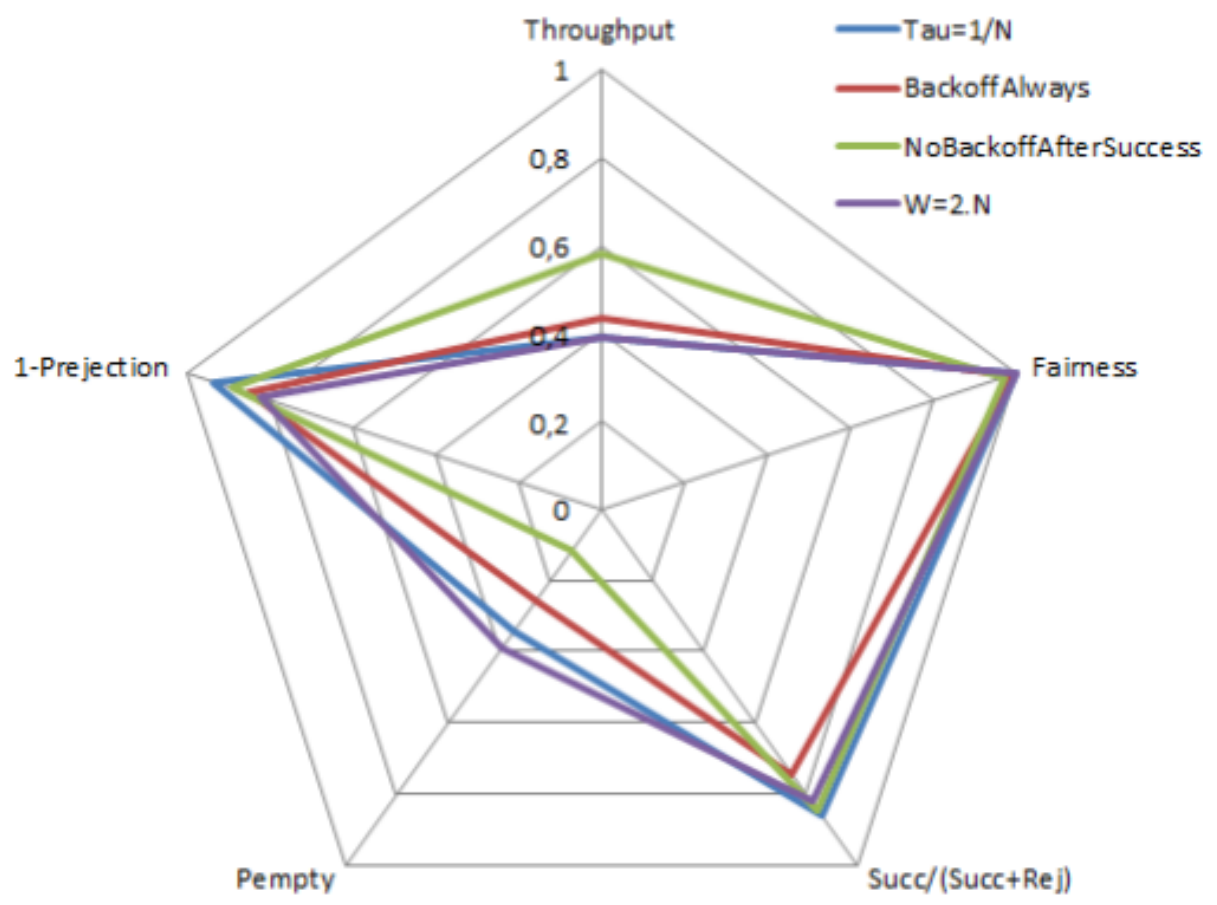

Fig. 12. Comparative performance evaluation for 4 nodes. 


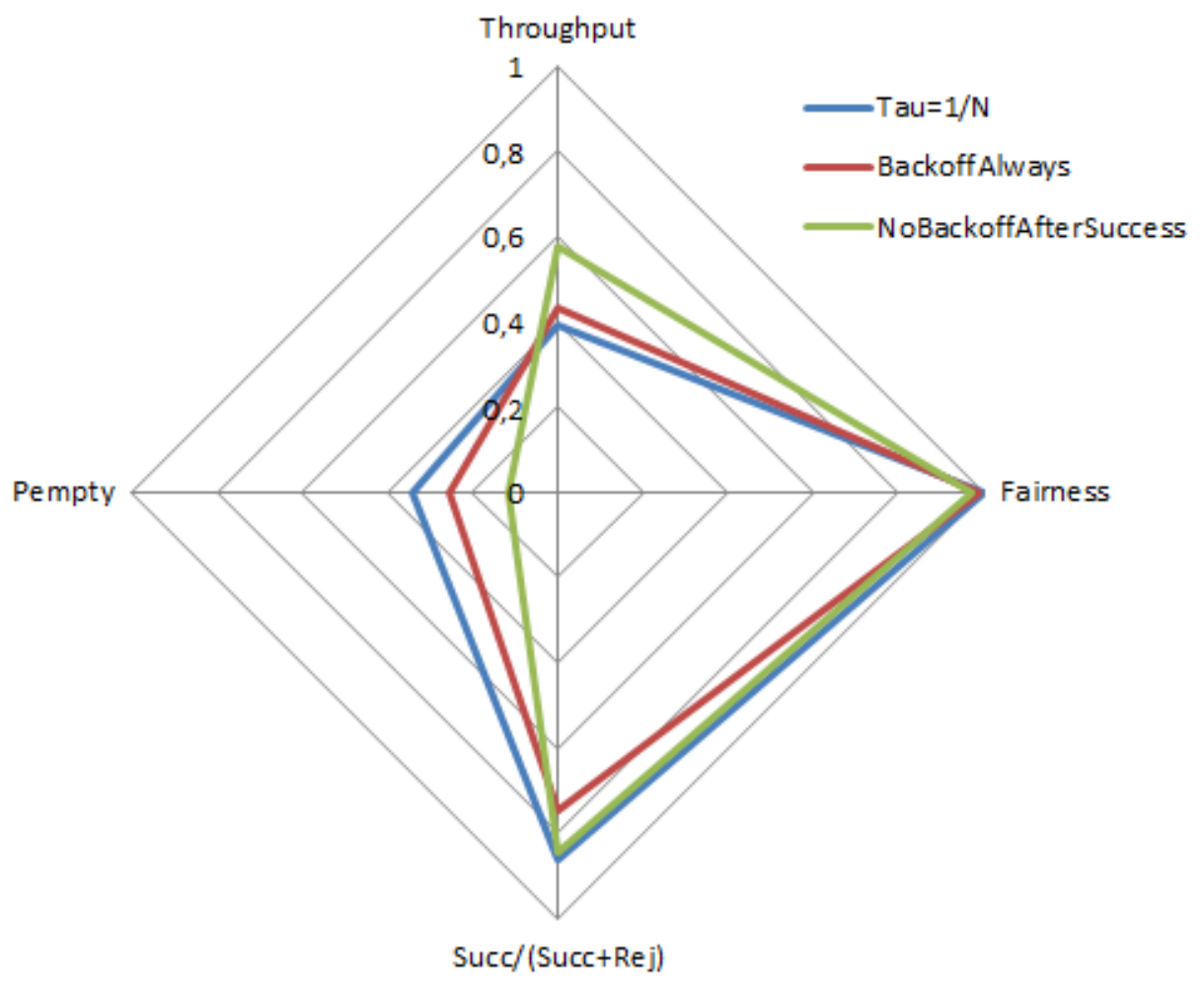

Fig. 13. Comparative performance evaluation for 8 nodes.

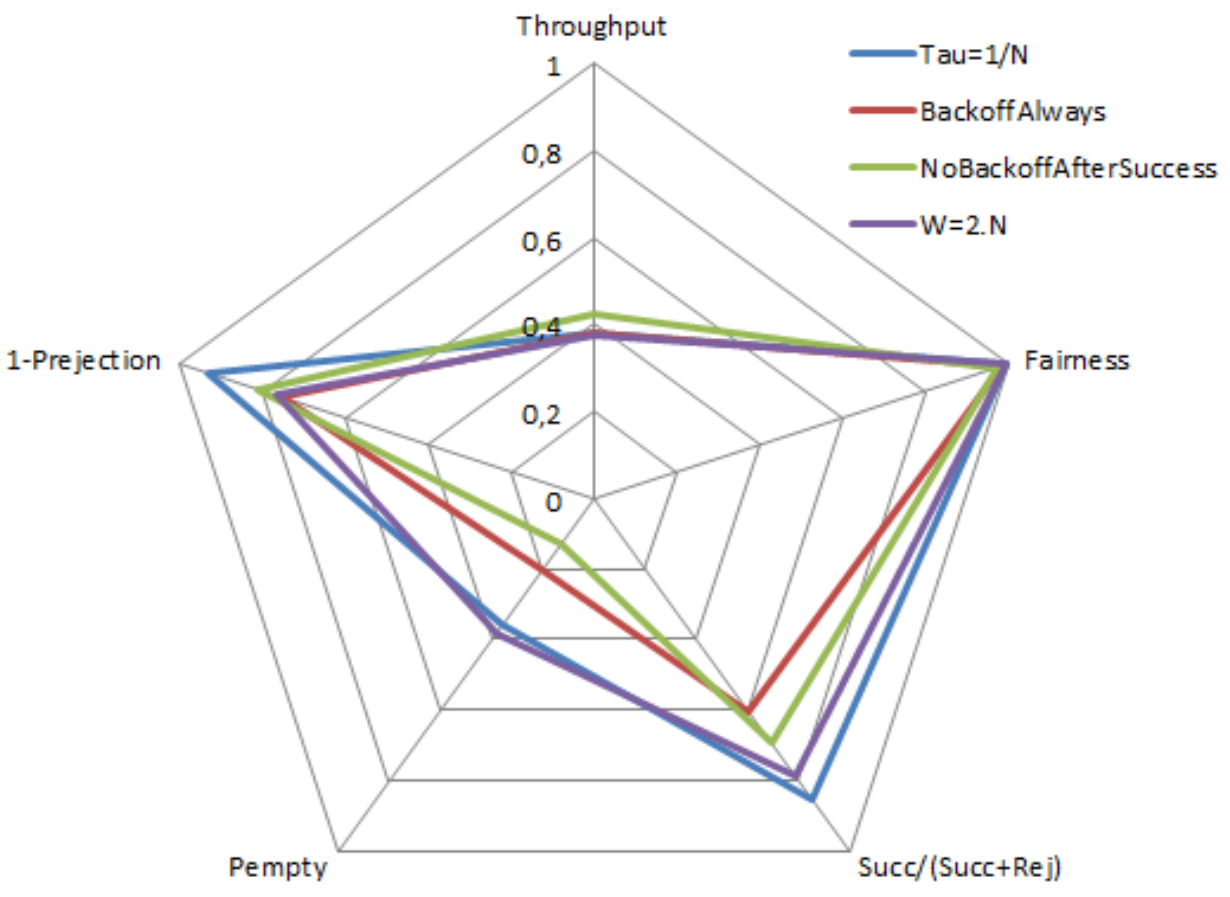

Fig. 14. Comparative performance evaluation for 16 nodes. 


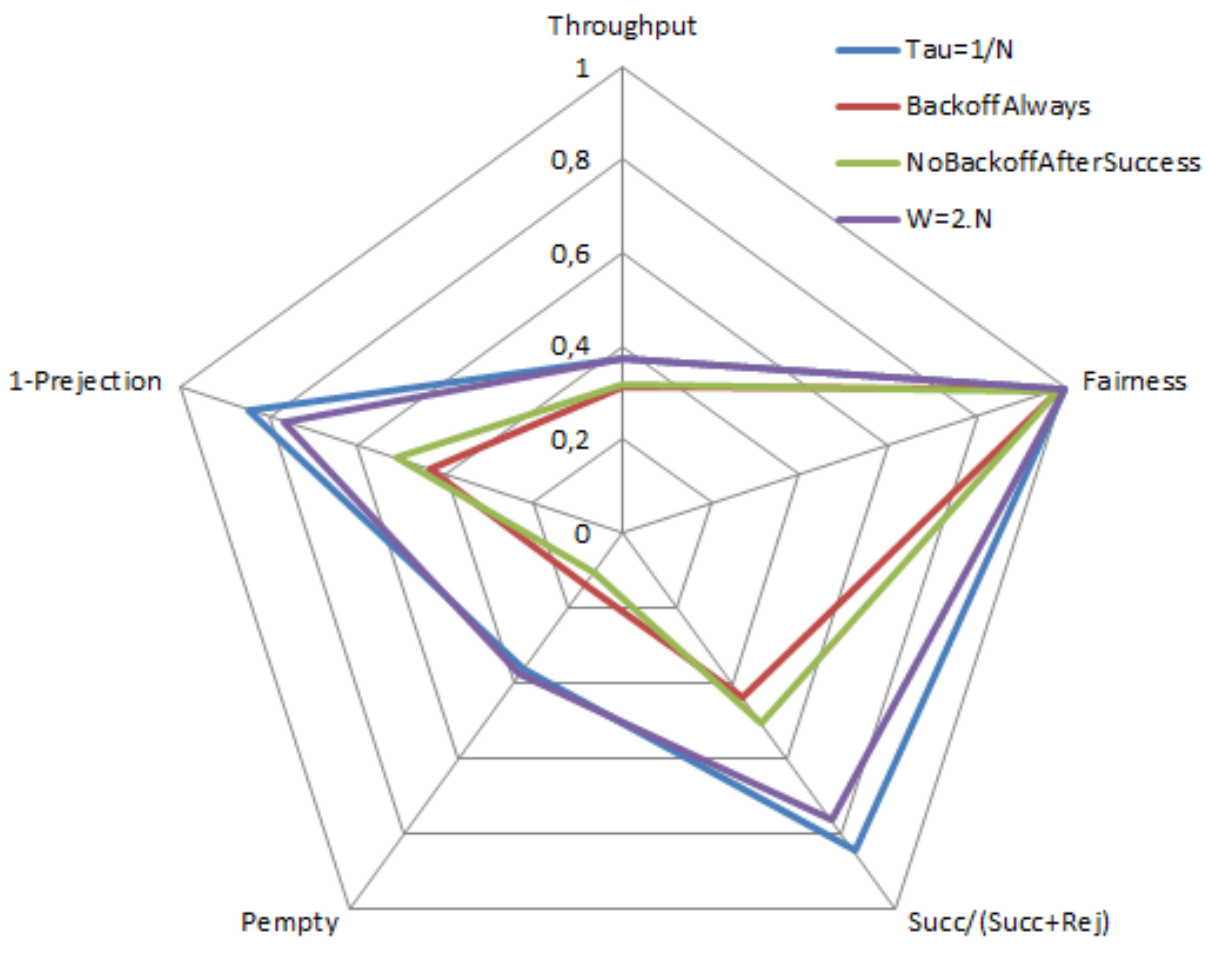

Fig. 15. Comparative performance evaluation for 32 nodes.

\section{CONCLUSION}

In this paper, we have analyzed two medium access protocols operating in a wireless slotted network. Their performances have been evaluated by means of two new Markov models that take into account the unsuccessful transmission of the previous message. Extensive simulations show a very good match between the model and the simulation results. For a moderate medium load, the protocol performing a backoff before each transmission outperforms the TSCH protocol, when the number of neighboring nodes is greater than or equal to 8. For a smaller number of neighboring nodes, the TSCH protocol provides a higher throughput. For a high medium load, the TSCH protocol provides the highest normalized throughput at the cost of some unfairness in the transmission opportunities.

An extension of this work could be to introduce a more accurate model of the physical layer, taking into account transmission decay, fading and capture phenomena. It would lead to a collision evaluation that is more representative of the real world.

\section{ACKNOWLEDGMENT}

Study co-funded by CNES and Inria in the framework of the CNES Launchers Research and Technology program.

\section{REFERENCES}

[1] F. Sebbak and F. Benhammadi, "Majority-consensus fusion approach for elderly iot-based healthcare applications," Annals of Telecommunications, vol. 72, no. 3, pp. 157-171, Apr 2017. [Online]. Available: https://doi.org/10.1007/s12243-016-0550-7

[2] M. Hadded, R. Zagrouba, P. Muhlethaler, and L. Saidane, "An adaptive TDMA slot assignment strategy in vehicular ad hoc network," Journal of Machine to Machine Communications, vol. 1, no. 2, pp. 175-194, Apr 2014. [Online]. Available: https://hal.archives-ouvertes.fr/hal-01263243

[3] Z. Doukha, S. A. BenMussa, K. Z. Ghafoor, I. Loumachi, and S. Moussaoui, "Load balancing aware sdma-based beaconing approach in vehicular ad hoc networks," Annals of Telecommunications, vol. 72, no. 3, pp. 189-197, Apr 2017. [Online]. Available: https://doi.org/10.1007/s12243-017-0562-y

[4] A. Derhab, M. Guerroumi, and M. Younis, "Wireless and mobile sensing technologies for the future internet," Annals of Telecommunications, vol. 72 , no. 3, pp. 117-118, Apr 2017. [Online]. Available: https://doi.org/10.1007/s12243-017-0570-y

[5] M. Hermann, T. Pentek, and B. Otto, "Design Principles for Industrie 4.0 Scenarios," in Proceedings of the 2016 49th Hawaii International Conference on System Sciences (HICSS), ser. HICSS '16. Washington, DC, USA: IEEE Computer Society, 2016, pp. 3928-3937. [Online]. Available: http://dx.doi.org/10.1109/HICSS.2016.488

[6] H. Kagermann and W. Wahlster, and J. Helbig, eds., "Recommendations for implementing the strategic initiative Industrie 4.0: Final report of the Industrie 4.0 working group," Frankfurt, Germany, Tech. Rep., 2013.

[7] IEEE SA, "IEEE Standard for Local and metropolitan area networks-Part 15.4: Low-Rate Wireless Personal Area Networks (LR-WPANs)," IEEE, IEEE Std 802.15.4-2011 (Revision of IEEE Std 802.15.4-2006), Sept 2011. [Online]. Available: http://dx.doi.org/10.1109/ieeestd.2006.232110

[8] IEEE, "IEEE Standard for Local and metropolitan area networks-Part 15.4: Low-Rate Wireless Personal Area Networks (LR-WPANs) Amendment 1: MAC sublayer," IEEE, IEEE Std 802.15.4e-2012 (Amendment to IEEE Std 802.15.4-2011), February 2012. [Online]. Available: http://standards.ieee.org/getieee802/download/802.15.4e-2012.pdf

[9] NS3-Consortium, "NS3 a discrete network event simulator for internet systems." [Online]. Available: https://www.nsnam.org/ 
[10] L. G. Roberts, "Aloha packet system with and without slots and capture," SIGCOMM Comput. Commun. Rev., vol. 5, no. 2, pp. 28-42, Apr. 1975. [Online]. Available: http://doi.acm.org/10.1145/1024916.1024920

[11] G. Bianchi, "Performance analysis of the IEEE 802.11 distributed coordination function," IEEE Journal on Selected Areas in Communications, vol. 18, no. 3, pp. 535-547, 2000

[12] I. E. Korbi and L. Saidane, "Analytical model of the earliest deadline first policy over 802.11," in Fourth Advanced International Conference on Telecommunications (AICT 2008), Athens, Greece, 2008, pp. 180-187.

[13] A. Bhattacharya and A. Kumar, "Analytical modeling of IEEE 802.11-Type CSMA/CA networks with short term unfairness," IEEE/ACM Transactions on Networking, vol. PP, no. 99, pp. 1-18, 2017. [Online]. Available: https://ieeexplore.ieee.org/document/8052230/

[14] T. R. Park, T. H. Kim, J. Y. Choi, S. S. Choi, and W. H. Kwon, "Throughput and energy consumption analysis of IEEE 802.15. 4 slotted CSMA/CA," Electronics Letters, vol. 41, no. 18, pp. 1017-1019, 2005.

[15] S. Pollin, M. Ergen, S. C. Ergen, B. Bougard, L. V. der Perre, I. Moerman, A. Bahai, P. Varaiya, and F. Catthoor, "Performance analysis of slotted carrier sense IEEE 802.15.4 medium access layer," IEEE Trans. Wireless Communications, vol. 7, no. 9, pp. 3359-3371, 2008. [Online]. Available: http://dx.doi.org/10.1109/TWC.2008.060057

[16] P. Park, P. D. Marco, P. Soldati, C. Fischione, and K. H. Johansson, "A Generalized Markov Chain Model For Effective Analysis of Slotted IEEE 802.15.4," in Mobile Adhoc and Sensor Systems (MASS). IEEE Computer Society, October 2009, pp. 130-139. [Online]. Available: http://dblp.uni-trier.de/db/conf/mass/mass2009.html\#ParkMSFJ09

[17] H. Wen, C. Lin, Z.-J. Chen, H. Yin, T. He, and E. Dutkiewicz, "An Improved Markov Model for IEEE 802.15.4 Slotted CSMA/CA Mechanism," Journal of Computer Science and Technology, vol. 24, no. 3, pp. 495-504, 2009. [Online]. Available: http://dx.doi.org/10.1007/s11390-009-9240-5

[18] R. Jain, D. Chiu, and W. Hawe, "A quantitative measure of fairness and discrimination for resource allocation in shared computer systems," CoRR, vol. cs.NI/9809099, pp. 1-35, 1998. [Online]. Available: http://arxiv.org/abs/cs.NI/9809099 